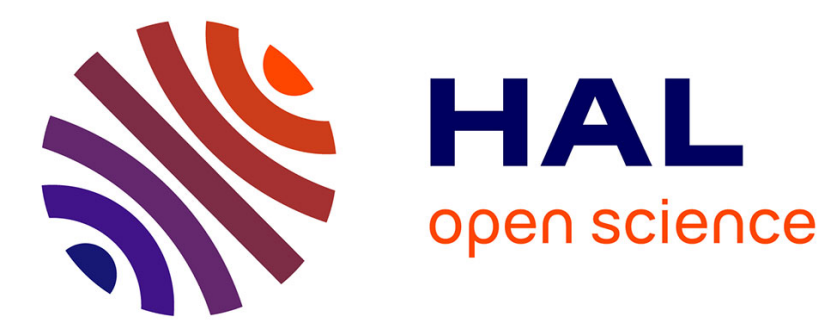

\title{
Performance of a linear robust control strategy on a nonlinear model of spatially developing flows
}

\author{
Eric Lauga, T.R. Bewley
}

\section{To cite this version:}

Eric Lauga, T.R. Bewley. Performance of a linear robust control strategy on a nonlinear model of spatially developing flows. Journal of Fluid Mechanics, 2004, 512 (august), pp.343-374. 10.1017/s0022112004009711 . hal-01024942

HAL Id: hal-01024942

https://hal-polytechnique.archives-ouvertes.fr/hal-01024942

Submitted on $30 \mathrm{Jul} 2014$

HAL is a multi-disciplinary open access archive for the deposit and dissemination of scientific research documents, whether they are published or not. The documents may come from teaching and research institutions in France or abroad, or from public or private research centers.
L'archive ouverte pluridisciplinaire HAL, est destinée au dépôt et à la diffusion de documents scientifiques de niveau recherche, publiés ou non, émanant des établissements d'enseignement et de recherche français ou étrangers, des laboratoires publics ou privés. 


\title{
Performance of a linear robust control strategy on a nonlinear model of spatially developing flows
}

\author{
By ERIC LAUGA ${ }^{1,2} \uparrow$ AND THOMAS R. BEWLEY \\ ${ }^{1}$ Flow Control Lab, Department of MAE, University of California San Diego, La Jolla, \\ CA 92093-0411, USA \\ ${ }^{2}$ LadHyX, École Polytechnique-CNRS, 91128 Palaiseau Cedex, France
}

(Received 28 April 2003 and in revised form 24 March 2004)

This paper investigates the control of self-excited oscillations in spatially developing flow systems such as jets and wakes using $\mathscr{H}_{\infty}$ control theory on a complex GinzburgLandau (CGL) model. The coefficients used in this one-dimensional equation, which serves as a simple model of the evolution of hydrodynamic instability waves, are those selected by Roussopoulos \& Monkewitz (Physica D 1996, vol. 97, p. 264) to model the behaviour of the near-wake of a circular cylinder. Based on noisy measurements at a point sensor typically located inside the cylinder wake, the compensator uses a linear $\mathscr{H}_{\infty}$ filter based on the CGL model to construct a state estimate. This estimate is then used to compute linear $\mathscr{H}_{\infty}$ control feedback at a point actuator location, which is typically located upstream of the sensor. The goal of the control scheme is to stabilize the system by minimizing a weighted average of the 'system response' and the 'control effort' while rigorously bounding the response of the controlled linear system to external disturbances. The application of such modern control and estimation rules stabilizes the linear CGL system at Reynolds numbers far above the critical Reynolds number $R e_{c} \approx 47$ at which linear global instability appears in the uncontrolled system. In so doing, many unstable modes of the uncontrolled CGL system are linearly stabilized by the single actuator/sensor pair and the model-based feedback control strategy. Further, the linear performance of the closed-loop system, in terms of the relevant transfer function norms quantifying the linear response of the controlled system to external disturbances, is substantially improved beyond that possible with the simple proportional measurement feedback proposed in previous studies. Above $R e \approx 84$, the $\mathscr{H}_{\infty}$ control designs significantly outperform the corresponding $\mathscr{H}_{2}$ control designs in terms of their ability to stabilize the CGL system in the presence of worst-case disturbances. The extension of these control and estimation rules to the nonlinear CGL system on its attractor (a simple limit cycle) stabilizes the full nonlinear system back to the stationary state at Reynolds numbers up to $R e \approx 97 \mathrm{using}$ a single actuator/sensor pair, fixed-gain linear feedback and an extended Kalman filter incorporating the system nolinearity.

\section{Introduction}

Understanding the physical mechanisms responsible for self-excited hydrodynamic phenomena, and how such self-excitation can best be subdued or eliminated by the

$\dagger$ Present address: Division of Engineering and Applied Sciences, Harvard University, Cambridge MA 02138, USA. 
action of control feedback, are problems of important engineering consequences. For example, periodic vortex shedding in the flow past a structure can lead to large oscillating forces on either the structure causing the shedding or other structures situated downstream. This can cause significant structural damage when the flow velocity is such that the natural frequency of the vortex shedding is near one of the resonant frequencies of the structure in question. This paper investigates the use of linear robust control theory and an illuminating model problem in order to shed light on some of the relevant issues in the feedback control of such phenomena.

Open shear flow instabilities are commonly interpreted using the concepts of absolute and convective instability (Huerre \& Monkewitz 1990; Huerre \& Rossi 1998). An unstable linear system is termed absolutely unstable if a localized impulse injection of energy spreads downstream and upstream and eventually disrupts the entire media; on the other hand, if the system relaxes to the equilibrium at any fixed location as the growing disturbance energy is advected downstream, it is termed convectively unstable. In the preferential frame of reference of the system (which can usually be defined without ambiguity, owing to the presence of bodies or inflow conditions), an important characterization of the system is the normal mode with vanishing group velocity with maximum growth rate, referred to as the absolute mode. If the growth rate of this mode is positive, the system is absolutely unstable and is described as a temporal instability; if it is negative, the system is convectively unstable and is described as a spatial instability. This criterion was first introduced in plasma physics and, together with the requirement that the absolute mode must arise from the coalescence of upstream and downstream spatially developing modes, is referred to as the Briggs-Bers criterion (Briggs 1964; Bers 1983).

In fluid mechanics, the definitions given above may strictly only be applied to parallel flows, for which the stability characteristics do not depend upon the streamwise coordinate. Nevertheless, the analysis can be extended locally at each streamwise location when the flow is only slowly diverging, i.e. when the length scale $\lambda$ of the typical instabilities is small with respect to the characteristic evolution length scale $L$ of the mean flow. This extension may be used to establish a valuable link between the spatially localized short-time response to local forcing and the global long-time response of the entire system (Huerre \& Monkewitz 1990; Monkewitz 1993) when control feedback is not applied. In this setting, the flow may be characterized by analysis of its local stability properties, obtained by artificially extending the local values of the flow characteristics to the entire system.

Flows which display only local convective instability or for which the size of the pocket of absolute instability is not sufficiently large will behave in the linear regime as noise amplifiers with extrinsic dynamic behaviour, returning to rest when external forcing on the system is removed and all disturbances wash downstream. Flat-plate boundary layers (Gaster 1968) and coflowing mixing layers (Huerre \& Monkewitz 1985) behave in this manner. Alternatively, certain marginally globally stable flows behave like slightly damped oscillators, in which an intrinsic oscillating mode is preferentially excited by system disturbances. Low-Mach-number axisymmetric jets (Huerre \& Monkewitz 1990) demonstrate this type of behaviour. Finally, flows in which a sufficiently large pocket of absolute instability is present typically behave as oscillators with intrinsic dynamic behaviour, sustaining growing (and eventually saturating) perturbations in the system even when all external forcing on the system is removed. Such is the case for capillary jets (Monkewitz 1990) and bluff-body wakes (Huerre \& Monkewitz 1990). In this case, as a global control parameter is increased (the Weber number in the case of capillary jets, the Reynolds number in the case 
of wakes) a supercritical Hopf bifurcation eventually takes place in which a stable steady solution bifurcates to a periodic limit cycle whose magnitude is governed by the nonlinearities of the system (Chomaz, Huerre \& Redekopp 1987, 1988; Couairon \& Chomaz 1999; Pier \& Huerre 2001). The unstable mode prevailing in the linear regime, termed the linear global mode, is a time-harmonic spatial mode that satisfies a streamwise eigenvalue problem; in the case of linear global instability, the system eventually saturates into a limit cycle, termed the nonlinear global mode (Pier et al. 1998; Pier \& Huerre 2001).

Our main interest here, the bluff-body wake, has been the central subject of many studies. In the early 1980s, the elegent experimental discoveries of Mathis, Provansal \& Boyer (1984) and Provansal, Mathis \& Boyer (1987) motivated renewed interest in this flow. These studies, perhaps the first studies of an open flow considered as a dynamical system, showed that the von Kármán shedding characteristic of bluff-body wakes was not a response to continuous excitations upstream, but was indeed a limitcycle oscillation of the near wake in which an initially exponentially growing linear global mode nonlinearly saturates. This was the first link between theoretical work on linear global instability and real flows, and has been used ever since as motivation for the study of both linear and nonlinear global instability. These results were related to local near-wake instability in Monkewitz (1988). For the case of the cylinder wake, when the Reynolds number based on the cylinder diameter, $R e$, exceeds $R e \approx 5$, a local pocket of convective instability appears in the near wake, this region growing in dimension when $R e$ is increased further. Upon reaching $R e \approx 25$, a second local bifurcation takes place and a region of absolutely unstable flow appears within the convectively unstable flow. The size of the absolutely unstable region increases with $R e$ up to the critical value $R e_{c} \approx 47$ at which point a global bifurcation takes place in the wake, and vortex shedding appears. Below this critical value, noise amplification takes place, but when all exterior forcing is removed, the system eventually comes back to rest everywhere. For further review of bluff-body-wake phenomena in the uncontrolled setting, especially of the three-dimensional effects that appear for $R e \gtrsim 190$, the reader is referred to Williamson (1996). The goal of the present study is to develop simple feedback control rules based on $\mathscr{H}_{\infty}$ control theory to delay the appearance of linear global instability until some $R e>47$.

As previously proposed (Ffowcs Williams \& Zhao 1989; Roussopoulos 1993), since vortex shedding is the nonlinear limit cycle of an initially linear instability, linear control rules suppressing linear instability might also prevent vortex shedding, possibly (at least at some Reynolds numbers) regulating to zero the fully nonlinear system from everywhere on the attracting limit cycle of the uncontrolled nonlinear system. The extrapolation of linear control strategies to the control of nonlinear systems is based on the hypothesis that, at a given Reynolds number, the domain of convergence to the stationary state in the linearly controlled nonlinear system includes the entire nonlinear attractor of the uncontrolled system. Whether or not linear feedback may be found such that this hypothesis is true in a particular system is often difficult to predict a priori, but may be at least partially verified by simulation. $\dagger$ In the control

$\dagger$ Note that Lyapunov-based analysis may sometimes be used to establish rigorously an (often conservative) lower bound on the domain of stability of a controlled nonlinear system. Using a constructive control design approach such as backstepping (which leverages Lyapunov-based analysis), it is even possible in certain cases to design globally stabilizing nonlinear feedback. An application of such an approach to the CGL equation is discussed by Aamo \& Krstic (2003). In situations for which such global stabilization is possible, synthesizing a control design which is both 
of fluid-mechanical systems, this hypothesis has sometimes proved to be true, as in the control of turbulent channel flow at very low Reynolds number as studied in Högberg, Bewley \& Henningson (2003). On the other hand, it has sometimes proved to be false, as in the control of a simple convection loop (Bewley 1999). In these two examples, the attractor of the uncontrolled nonlinear system is, in fact, chaotic, and the nonlinear concept of gain scheduling (that is, selecting linear feedback gains based on some measure of the state of the system) has proved to be useful. In the present case, the attractor of the uncontrolled nonlinear system (that is, the vortex shedding) is a simple limit cycle. In open flows dominated by the effects of convection, such as the present, the idea of extrapolating linear control strategies to the full nonlinear system is particularly appealing, as far enough upstream in such systems, all perturbations (even those on the attractor of the full nonlinear system) are small, and thus linear analysis without gain scheduling is valid. Therefore, if the actuator and sensor are located sufficiently far upstream, linear control should have a stabilizing effect on the nonlinear synchronized behaviour.

As a model for spatially developing flows, the complex Ginzburg-Landau (CGL) equation has been chosen in this study. In previous studies, this one-dimensional model equation has proved to be quite useful for determining global frequency criteria (in both the linear (Chomaz et al. 1991) and nonlinear (Pier et al. 1998) regimes) which extend accurately to the full three-dimensional system governed by the Navier-Stokes equation (Monkewitz, Huerre \& Chomaz 1993; Pier \& Huerre 2001; Pier 2002). Moreover, because the eigenfunctions of the CGL model at sufficiently low $R e$ accurately represent all of the unstable linear global modes of the full Navier-Stokes system, analysis of this model equation has also allowed semi-quantitative predictions of the effect of proportional feedback control in physical experiments (Monkewitz 1989, 1993; Monkewitz et al. 1993; Roussopoulos \& Monkewitz 1996) of spatially developing flow systems.

A few previous investigations have studied the control of the present system using strategies based on physical insight or inspired by simple practical considerations. Using a linear CGL model, it was shown in Monkewitz (1989) that stabilization of self-excited oscillations with proportional measurement feedback from a single sensor to a single actuator was only effective near the global bifurcation point and for small values of the feedback gain. When the bifurcation parameter exceeded this narrow window, the feedback still stabilized the primary unstable mode, but it destabilized a secondary mode. In a related study, Park, Ladd \& Hendricks (1993) obtained similar conclusions. More recently, Roussopoulos \& Monkewitz (1996, hereinafter referred to as RM96) used a cylinder wake model, combining the usual linear streamwise CGL equation with a nonlinear diffusive spanwise CGL equation in order to account for both two- and three-dimensional effects. To control this model, they applied proportional measurement feedback, with the result that vortex shedding at $R e=50$ could only be suppressed in the spanwise vicinity of the sensor, even if the actuator acted uniformly over the entire span; this confirmed previous experimental results from Roussopoulos (1993). The model we will use in this paper is the two-dimensional version of the model used in RM96.

locally $\mathscr{H}_{\infty}$ optimal and globally (nonlinearly) stabilizing is possible, using the technique developed by Ezal, Pan \& Kokotovic (2000). For brevity, the present paper focuses on the development of linear $\mathscr{H}_{\infty}$-optimal control feedback; the possible synthesis of these linear control designs with globally stabilizing nonlinear control designs (in those limited cases for which this is currently possible) is deferred to future work. 
An earlier study (Lauga \& Bewley 2003) considered the full-information feedback control of the linear CGL model and the gradual decay of linear stabilizability of this system as the Reynolds number is increased. It was found that, even though in theory the system considered is linearly stabilizable for all $R e$, the control authority on the unstable modes becomes exponentially small as the Reynolds number is increased. For a given actuator position, an effective upper bound on the Reynolds number is reached above which numerical codes fail to determine a stabilizing feedback control rule via solution of the relevant Riccati equations. However, this apparent 'limit' is nothing more than a persistent numerical artefact, as it is strongly dependent on the numerical precision used in the computation of the feedback gains. Thus, such 'limits' do not represent fundamental features of the system considered. This study, in fact, motivates us to explore different measures to quantify the control system effectiveness in both the linear and nonlinear regimes, as we set forth to do in the present paper.

Finally, experimental and numerical studies of the control of vortex shedding have been the focus of several investigations. Proportional measurement feedback control of vortex shedding behind an oblong cylinder was implemented experimentally in Berger (1967) with success for a short range of Reynolds numbers, between $R e_{c}=79.2$ and $R e=90.3$. Since then, many other publications have considered various passive and physically based active strategies, either numerically (Park, Ladd \& Hendricks 1994; Min \& Choi 1999), theoretically (Monkewitz 1993; Park 1994), or experimentally (Roussopoulos 1993). In the comprehensive numerical study by Park et al. (1994), proportional measurement feedback control could suppress vortex shedding at $R e=60$ for select sensor locations, but at $R e=80$ the wake could not be controlled, as the primary vortex-shedding mode was stabilized, but a secondary shedding mode was not. This effect was also observed experimentally by Roussopoulos (1993). More recently, Min \& Choi (1999) used 'suboptimal' control theory (i.e. finitehorizon model predictive control theory applied over an infinitesimal time horizon) to completely stabilize vortex shedding in simulations up to $R e=160$. This impressive performance greatly exceeds previous results and motivates the present work, which attempts to stabilize a model of the wake using the linear $\mathscr{H}_{2} / \mathscr{H}_{\infty}$ control approach and time-independent feedback gains in a single-input single-output configuration for Reynolds numbers up to the onset of three-dimensional effects.

The present paper is organized as follows. In $\S 2$, we develop the control-oriented system model, presenting both the CGL equation and the spatial discretization used to represent this equation in state-space form. We briefly review in $\S 3$ the $\mathscr{H}_{2} / \mathscr{H}_{\infty}$ control theory used in the present work, the special considerations involved in applying this theory to the discretized CGL model, and the tools used to analyse its effectiveness. Section 4 is devoted to the study of the performance of the controlled linear system, including a comparison between modern and proportional control and a comparison of the effectiveness of the $\mathscr{H}_{2}$ and $\mathscr{H}_{\infty}$ control design strategies. Section 5 addresses the effectiveness of the linear control and estimation strategies on the nonlinear CGL system. Section 6 presents some concluding remarks.

\section{System modelling}

\subsection{The complex Ginzburg-Landau equation}

As stated in the introduction, the one-dimensional complex Ginzburg-Landau (CGL) equation was chosen in this study as a model of the evolution of instability waves in flows which develop slowly in the spatial coordinate $x$. The generic form of the CGL equation describes the spatio-temporal evolution of a complex streamfunction 
$\psi(x, t)$ of a perturbation wavepacket to some base-flow streamfunction $\Psi(X, t)$ close to marginal stability. It is given by

$$
\frac{\partial \psi}{\partial t}=-\kappa(X) \frac{\partial \psi}{\partial x}+\mu(X) \psi+v(X) \frac{\partial^{2} \psi}{\partial x^{2}}-\eta(X)|\psi|^{2} \psi
$$

where the complex coefficients $\kappa, \mu, v$ and $\eta$ are said to depend on the 'slow' variable $X=\epsilon x$ where $\epsilon \ll 1$, reflecting a weak spatial dependence of the base state on $x$. This equation parameterizes a combination of (or 'competition' between) advection, instability, diffusion and nonlinear effects on perturbations of the flow. Note that, in physical terms, $x$ is normalized by cylinder diameter $D$ and $t$ is normalized by $D$ over the free-stream velocity $U_{\infty}$.

Early work on the CGL equation can be found in Newell \& Whitehead (1969) in the case of Rayleigh-Bénard convection and Stewartson \& Stuart (1971) in the case of plane Poiseuille flow. An exact derivation of (2.1) in the context of weakly non-parallel shear flows such as jets or bluff-body wakes can be found in Monkewitz et al. (1993) (see also Huerre \& Monkewitz 1990; Le Gal et al. 2003).

Physical arguments can also be invoked to derive the CGL equation. A local normal mode stability analysis of a base state which depends weakly on the spatial coordinate $x$ can provide, at each location $x$, an absolute mode whose complex frequency is denoted by $\omega_{0}(x)$. The real part of $\omega_{0}$ is the temporal frequency of this mode, and the imaginary part of $\omega_{0}$ is the temporal growth rate of this mode. The results of Chomaz et al. (1991) can then be used to relate the global stability characteristics of a nonparallel slowly diverging uncontrolled system to its local characterizations given by $\omega_{0}(x)$. To achieve such a global characterization, the complex-valued function $\omega_{0}(x)$ is analytically continued into the complex plane $z$ defined such that $x=\operatorname{Re}(z)$. The closest point to the real axis in the complex plane for which $\mathrm{d} \omega_{0} / \mathrm{d} z=0$ is referred to as the wavemaker $z^{t}$. The value of $\omega_{0}$ at this complex turning point, $\omega^{t}=\omega_{0}\left(z^{t}\right)$, is (to first order in $\epsilon$ ) the complex global frequency of the entire uncontrolled linear system, which is linearly globally unstable if and only if $\operatorname{Im}\left(\omega^{t}\right)>0$. (Note that when a feedback control loop is used to relate sensing at one point in this system to forcing at another point, this analysis technique breaks down, and the global behaviour of the system must be characterized with different tools. The present paper presents appropriate tools to analyse the global dynamics of such a system in the closed-loop setting.)

As the linear response of the uncontrolled system to an impulse injection of energy will eventually be dominated by the single global mode $\left\{k_{0}(x), \omega_{0}(x)\right\}$ with zero group velocity $\partial \omega / \partial k=0$, the dispersion relation describing $\omega(k ; x)$ for a perturbation wavepacket to the base configuration may be approximated in the neighbourhood of this mode by a truncated Taylor series expansion in $k$ of the form

$$
\omega(k ; x) \approx \omega_{0}(x)+\frac{1}{2} \omega_{k k}(x)\left[k-k_{0}(x)\right]^{2} .
$$

As the behaviour of the system is dominated by the effect of this mode, we may proceed by assuming equality in this relation for all $\omega, k$ and $x$. Multiplying this equation by $\mathrm{i} \hat{\psi}_{\omega, k}(x)$ and inverse transforming the result with respect to both time $t$ and the spatial coordinate $x$, assuming that both the Fourier modes $\hat{\psi}_{\omega, k}(x)$ and the coefficients $\omega_{0}(x), \omega_{k k}(x)$ and $k_{0}(x)$ vary only slowly with $x$ and thus at any particular value of $x$ may be considered essentially invariant in $x$, leads to the CGL 
equation (2.1) for $\psi(x, t)=\sum_{\omega, k} \hat{\psi}_{\omega, k}(x) \mathrm{e}^{\mathrm{i} k x} \mathrm{e}^{-\mathrm{i} \omega t}$, linearized around the state $\psi=0$ :

$$
\frac{\partial \psi}{\partial t} \approx-\mathrm{i}\left[\omega_{0}(x)+\frac{1}{2} \omega_{k k}(x) k_{0}^{2}(x)\right] \psi+\omega_{k k}(x) k_{0}(x) \frac{\partial \psi}{\partial x}+\frac{1}{2} \mathrm{i} \omega_{k k}(x) \frac{\partial^{2} \psi}{\partial x^{2}} \triangleq \mathscr{L} \psi .
$$

For the purpose of analysis, we endow this equation with vanishing boudary conditions as $|x| \rightarrow \infty$. As a consequence, we restrict our attention to systems for which local instabilities are not dominated by the effects of boundaries, which is a valid approximation for many flows of physical interest, such as bluff-body wakes (Huerre \& Monkewitz 1990; Monkewitz et al. 1993; Shumm, Berger \& Monkewitz 1994; Hammond \& Redekopp 1997) and geophysical shear flows.

For the specific case of the wake behind a cylinder, recalling that $\mathrm{d} \omega_{0} / \mathrm{d} z=0$ at the wavemaker $z^{t}$, we expand the absolute mode $\left\{k_{0}(x), \omega_{0}(x)\right\}$ on the real line near the (complex) point $z^{t}$ and retain the minimal number of terms consistent with numerical simulations and experimental observations of bluff-body wake dynamics (see Monkewitz 1988; RM96):

$$
\left.\begin{array}{rl}
\omega_{0}(x) & =\omega^{t}+\frac{1}{2} \omega_{z z}^{t}\left(x-z^{t}\right)^{2}, \\
k_{0}(x) & =k^{t}+k_{z}^{t}\left(x-z^{t}\right), \\
\omega_{k k}(x) & =\omega_{k k}^{t} .
\end{array}\right\}
$$

Inserting these low-order expansions of the absolute mode and its derivatives into (2.3), we obtain an $x$-dependant model equation whose eigenmodes can be computed analytically in terms of Hermite polynomials and exponential functions (Chomaz et al. 1987). Note that the model is strictly valid only in the vicinity of the wavemaker (see Monkewitz et al. 1993 for more details); however, for weakly non-parallel base flows, the range of validity of this model includes a rather long streamwise extent around $\operatorname{Re}\left(z^{t}\right)$, within which we will assume the sensor and actuator are located.

The final step in modelling the unforced system consists of accounting for the bifurcation parameter (in the present case, the Reynolds number) that transforms the global behaviour of the system from time-damped to self-excited. Near the global bifurcation point $R e_{c}=47$, it is appropriate to perform a Taylor series expansion in powers of $\left(R e-R e_{c}\right)$ for each of the coefficients of the expansion given in (2.4). The simplest form of these $R e$ expansions consistent with experimental and numerical data, and appropriate numerical values of the coefficients for the present system (from RM96), are given by:

$$
\left.\begin{array}{rl}
\omega^{t} & =0.690+0.080 \mathrm{i}+10^{-3}(-1.59+4.47 \mathrm{i})\left(R e-R e_{c}\right), \\
\omega_{z z}^{t} & =0.108-0.057 \mathrm{i}, \\
z^{t} & =1.183-0.031 \mathrm{i}, \\
k^{t} & =1.452-0.844 \mathrm{i}+10^{-2}(0.341+1.1 \mathrm{i})\left(R e-R e_{c}\right), \\
k_{z}^{t} & =0.164-0.006 \mathrm{i}, \\
\omega_{k k}^{t} & =-0.292 \mathrm{i} .
\end{array}\right\}
$$

Figure 1 displays the local instability characteristics of the linear CGL model (2.3) (used in $\S 4$ of this paper) with the coefficients given in (2.4) and (2.5) for various values of the Reynolds number. The additional coefficient $\eta$ of the nonlinear term in (2.1) (used in $\S 5$ of this paper) models the Hopf bifurcation arising near $R e_{c}=47$ and the resulting nonlinear saturated state. As in RM96, we take its value as $\eta=0.0225-0.0671 \mathrm{i}$. Even though this model is, strictly speaking, derived for $R e$ in the vicinity of the threshold $R e_{c}$ and in the vicinity of the 'wavemaker' (the complex 


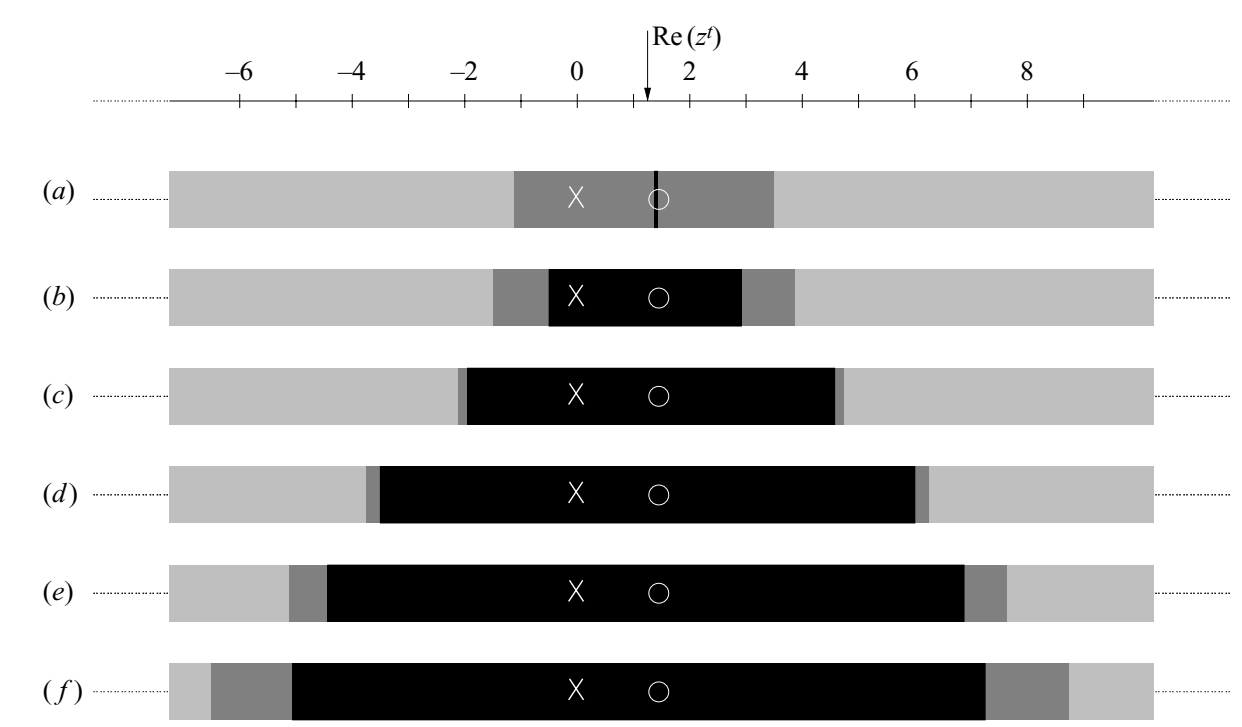

FIGURE 1. Sketches of the regions on the real $x$-axis of local stability (light grey), convective instability (dark grey) and absolute instability (black) of the uncontrolled linear wake model for: ( a $) R e=29$, onset of local absolute instability at $x=1.24,(b) R e=47$ onset of linear global instability, (c) $R e=100,(d) R e=175,(e) R e=235,(f) R e=284$. Representative locations for the sensor and actuator are marked with $\bigcirc$ and $\times$, respectively.

turning point $z^{t}$ mentioned previously), it has been shown for a wide variety of flows (Rayleigh-Bénard convection, Taylor-Couette flow, parallel wakes) that CGL-type models often remain accurate far outside this vicinity (Williamson 1996; Hammond \& Redekopp 1997; Couairon \& Chomaz 1999). We therefore approach the stabilization of this model system as far more than simply an academic exercise, hoping to obtain significant physical insight by considering it even for $R e$ far above the critical value $R e_{c}$.

\subsection{Spatial discretization and disturbance modelling}

The generic goal of control design is to determine appropriate inputs for a dynamic system with unknown disturbances and modelling errors, often coordinating such inputs with available noisy measurements of the system state, in order to achieve a specified objective. The objective of the present linear control design is to stabilize robustly the linearized CGL cylinder wake model. To achieve this objective, we will modify our linear model equation (2.3) by forcing it with both control and disturbance inputs and construct an equation modelling sensor measurements $y$ at some location $x_{s}$ in the following fashion:

$$
\left.\begin{array}{rl}
\frac{\partial \psi}{\partial t} & =\mathscr{L} \psi+w_{\psi}(x, t)+\delta\left(x-x_{f}\right) f(t), \\
y & =\psi\left(x_{s}\right)+w_{y}(t) .
\end{array}\right\}
$$

The input $f$ represents the strength of the control concentrated at a specific streamwise forcing location $x_{f}$. The value $x_{f}=0$, from RM96, was chosen for this study. It corresponds to actuation applied uniformly in the spanwise coordinate via, e.g. spanwise oscillation of the cylinder itself. The input $w_{\psi}$ accounts for process noise, state disturbances, and modelling errors. The output $y$ is the measurement at the specific streamwise location $x_{s}$ from a sensor corrupted by a finite amount of 
measurement noise $w_{y}$. The value $x_{s}=1.5$, from RM96, was chosen for this study. It corresponds to a sensor located 1.5 diameters downstream of the body on the symmetry axis. Flow perturbations are assumed to be two-dimensional.

Linear control theory is well developed for systems in standard state-space form

$$
\left.\begin{array}{l}
\dot{\boldsymbol{x}}=\boldsymbol{A} \boldsymbol{x}+\mathbf{G}_{1} \boldsymbol{w}_{1}+\mathbf{B} \boldsymbol{u} \\
\boldsymbol{y}=\boldsymbol{C} \boldsymbol{x}+\alpha \mathbf{G}_{2} \boldsymbol{w}_{2}
\end{array}\right\}
$$

where $\boldsymbol{x}$ is the state vector, $\boldsymbol{w}_{1}$ is the state disturbance vector, $\boldsymbol{u}$ is the control vector, $\boldsymbol{y}$ is the measurement vector, and $\boldsymbol{w}_{2}$ is the measurement noise vector. We will therefore discretize the CGL partial differential equation, (2.6), and express it as a linear ordinary differential equation of this form. A standard Fourier collocation technique on a finite domain is used, with a grid stretching function given by

$$
x_{n}=\xi_{n}-E\left\{\tanh \left[\frac{\xi_{n}-\xi_{f}}{e}\right]+\tanh \left[\frac{\xi_{n}-\xi_{s}}{e}\right]+\tanh \left[\frac{\xi_{s}}{e}\right]\right\},
$$

with the $\xi_{n}$ variable uniformly distributed on $\left(\xi_{\text {inf }}, \xi_{\text {sup }}\right)$. With this formulation, the two stretching parameters $E$ and $e$ facilitate the clustering of gridpoints in the vicinity of the actuator and sensor in order to resolve the significant effects of the pointwise forcing and sensing on the closed-loop system dynamics, while the grid smoothly returns to uniform away from these locations; the values $E=2.87$ and $e=3$ were found to be adequate in this regard in the present simulations. The parameters $\xi_{f}$ and $\xi_{s}$ are selected to cluster gridpoints near the physical forcing location $x_{f}=0$ and sensor location $x_{s}=1.5$; this is achieved by selecting $\xi_{f}=0$ and solving the equation $1.5=\xi_{s}-2 E \tanh \left(\xi_{s} / e\right)$ for $\xi_{s}$, resulting in $\xi_{s}=7.143$. By selecting $\xi_{\text {inf }}=-15.7$ and $\xi_{\text {sup }}=24$ and defining $N=140$ gridpoints via this approach, adequate resolution is achieved to resolve the present system dynamics. The field $\psi$ representing the dynamics of the perturbations is discretized as the state vector $\boldsymbol{x}$ (not to be confused with the spatial coordinate $x$ ) containing the state values at the gridpoints inside the domain considered. The state is prescribed to be periodic, though the domain size used is large enough for the state to approach zero well before the edge of the computational domain. All results presented here have been verified to be independent of grid resolution, box size, grid stretching and the spatial discretization method (an eighth-order central finite-difference scheme with homogeneous boundary conditions was also implemented for comparison).

The vectors comprising the state-space representation (2.7) are the vectors $\boldsymbol{x}$ and $\boldsymbol{w}_{1}$ (discretization of $\psi$ and $w_{\psi}$, respectively, on the grid points $\left\{x_{1}, \ldots, x_{N}\right\}$ ) and the scalars $\boldsymbol{u}=(f), \boldsymbol{y}=(y), \boldsymbol{w}_{2}=\left(w_{y}\right)$. The matrix $\boldsymbol{A}$ is determined by spatial discretization of the operator $\mathscr{L}$ with standard Fourier collocation techniques on the stretched grid. The control, applied at the gridpoint $x_{\lambda}$, and the measurement, taken at the gridpoint $x_{\kappa}$, are incorporated into the state-space representation by defining

$$
(\boldsymbol{B})_{i 1}=\frac{2}{x_{\lambda+1}-x_{\lambda-1}} \delta_{i \lambda}, \quad(\boldsymbol{C})_{1 j}=\delta_{j \kappa},
$$

where $\delta_{i j}$ denotes the Kronecker delta. Disturbances are modelled by defining $\mathbf{G}_{1}$ and $\alpha \mathbf{G}_{2}$ as the square root of any known or expected covariance structure of the state disturbances and the measurement noise, respectively. The scalar $\alpha^{2}$ is identified as an adjustable parameter which defines the ratio of the maximum singular value of the covariance of the measurement noise divided by the maximum singular value of the covariance of the state disturbances; without loss of generality, we take $\bar{\sigma}\left(\mathbf{G}_{1}\right)=\bar{\sigma}\left(\mathbf{G}_{2}\right)=1$. The matrix $\mathbf{G}_{1}$ effectively reflects which state disturbances are 
assumed to be strongest. As the measurement in the present system is just a scalar, we define $\boldsymbol{G}_{2}=1$. Small $\alpha$ implies relatively high overall confidence in the measurements, whereas large $\alpha$ implies relatively low overall confidence in the measurements. Note that, for the present simulations (for lack of any better disturbance modelling) we have assumed $\boldsymbol{G}_{1}=\boldsymbol{I}$. Other choices should be studied in future investigations by more closely modelling the disturbance covariance of interest in physical systems and their sensitivity to external disturbances.

\section{Control design and analysis tools}

\subsection{Control objective}

A cost function $\mathscr{J}$ defining the infinite-horizon control problem at hand is now specified that weighs together the state $\boldsymbol{x}$, the (scalar) control $\boldsymbol{u}$, and the disturbances $w$ such that

$$
\mathscr{J} \triangleq \mathscr{E}\left[\boldsymbol{x}^{*} \boldsymbol{Q} \boldsymbol{x}+\ell^{2} \boldsymbol{u}^{*} \boldsymbol{R} \boldsymbol{u}-\gamma^{2} \boldsymbol{w}^{*} \boldsymbol{S} \boldsymbol{w}\right], \quad \boldsymbol{w}=\left(\begin{array}{l}
\boldsymbol{w}_{1} \\
\boldsymbol{w}_{2}
\end{array}\right), \quad \boldsymbol{S}=\left(\begin{array}{ll}
\boldsymbol{S}_{1} & 0 \\
0 & \boldsymbol{S}_{2}
\end{array}\right),
$$

where we take the expectation $\mathscr{E}$ to be defined by $\mathscr{E}[\cdot]=\lim _{T \rightarrow \infty}(1 / T) \int_{0}^{T}[\cdot] \mathrm{d} t$. It is assumed that $\boldsymbol{Q} \geqslant 0, \boldsymbol{R}>0$ and $\boldsymbol{S}>0$. The matrix $\boldsymbol{Q}$ shaping the dependence on the state in the cost function, $\boldsymbol{x}^{*} \boldsymbol{Q} \boldsymbol{x}$, may be selected to approximate numerically any of a variety of physical properties of the flow system. In the present case, we define a grid-independent metric based on the mean-square value of $\psi$ over the domain of interest:

$$
Q_{i j}= \begin{cases}x_{2}-x_{1} & \text { for } i=j=1, \\ x_{N}-x_{N-1} & \text { for } i=j=N, \quad \Rightarrow \quad \boldsymbol{x}^{*} \mathbf{Q} \boldsymbol{x} \approx \int_{\mathbb{R}}|\psi|^{2} \mathrm{~d} x . \\ \delta_{i j} \frac{x_{i+1}-x_{i-1}}{2} & \text { otherwise, }\end{cases}
$$

A variety of other metrics might also be tried in the future. We note that the control objective selected here targets all components of the state, not only the measured components. In order to retain an equivalent control formulation upon refinement of the numerical grid, $\boldsymbol{R}$ and $\boldsymbol{S}$ should also be chosen carefully. In the present formulation, we take $\boldsymbol{R}=1, \boldsymbol{S}_{1}=\mathbf{Q}$, and $\boldsymbol{S}_{2}=1$. Note that if we had taken $\boldsymbol{S}_{1}=\boldsymbol{I}$, the control problem would change whenever the numerical grid is modified, significantly hindering physical interpretation of the results. With the present strategy, as the grid is refined, the discrete matrices representing the feedback gains (denoted by $\boldsymbol{K}$ and $\boldsymbol{L}$ in the following section) converge to continuous weighting functions, and the definitions of the tunable parameters $\ell, \alpha$ and $\gamma$ in the control formulation remain fixed.

\subsection{The structure of the compensator}

As only a limited number of noisy measurements $\boldsymbol{y}$ of the state $\boldsymbol{x}$ are available in any practical control implementation, it is beneficial to develop a filter which extracts as much useful information as possible from the available flow measurements before using this filtered information to compute a suitable control. In modern control theory, a model of the system itself is used as this filter, and the filtered information extracted from the measurements is simply an estimate of the state of the physical system. By modeling the influence of the unknown disturbances in (2.7), the system 
model takes the form

$$
\begin{aligned}
\dot{\hat{\boldsymbol{x}}} & =\boldsymbol{A} \hat{\boldsymbol{x}}+\mathbf{G}_{1} \hat{\boldsymbol{w}}_{1}+\boldsymbol{B} \boldsymbol{u}-\boldsymbol{v}, \\
\hat{\boldsymbol{y}} & =\boldsymbol{C} \hat{\boldsymbol{x}}+\alpha \mathbf{G}_{2} \hat{\boldsymbol{w}}_{2},
\end{aligned}
$$

where $\hat{\boldsymbol{x}}$ is the state estimate, $\hat{\boldsymbol{w}}_{1}$ is a state disturbance estimate, $\hat{\boldsymbol{w}}_{2}$ is a measurement noise estimate, and $\boldsymbol{v}$ is a feedback term based on the difference between the measurement of the state $\boldsymbol{y}$ and the corresponding quantity in the model $\hat{\boldsymbol{y}}$ such that

$$
\boldsymbol{v}=\boldsymbol{L}(\boldsymbol{y}-\hat{\boldsymbol{y}}) \text {. }
$$

The control $\boldsymbol{u}$, in turn, is based on the state estimate $\hat{\boldsymbol{x}}$ such that

$$
\boldsymbol{u}=\boldsymbol{K} \hat{\boldsymbol{x}} .
$$

Equation (2.7) is referred to as the 'plant', (3.3) is referred to as the 'estimator', and (3.4) is referred to as the 'controller'. The estimator (3.3) and the controller (3.4), taken together, will be referred to as the 'compensator'. The problem at hand is to compute linear time-invariant matrices $\boldsymbol{K}$ and $\boldsymbol{L}$ and some estimate of the disturbance, $\hat{\boldsymbol{w}}$, such that: (i) the estimator feedback $\boldsymbol{v}$ forces $\hat{\boldsymbol{x}}$ towards $\boldsymbol{x}$ (that is, $\hat{\boldsymbol{x}}$ becomes an accurate estimate of the unknown state $\boldsymbol{x}$ ), and (ii) the controller feedback $\boldsymbol{u}$ forces $\boldsymbol{x}$ towards zero (that is, the unstable modes of the system are stabilized).

\subsection{The $\mathscr{H}_{\infty}$ control solution}

Given the structure of the system defined in (2.7), (3.3) and (3.4) and the control objective defined in (3.1), the $\mathscr{H}_{\infty}$ compensator is determined by simultaneously minimizing the cost function $\mathscr{J}$ with respect to the control $\boldsymbol{u}$ and maximizing $\mathscr{J}$ with respect to the disturbance $\boldsymbol{w}$. In such a way, a control $\boldsymbol{u}$ is found which maximally attains the control objective even in the presence of a disturbance $w$ which maximally disrupts this objective. For sufficiently large values of $\gamma$ and for a system which is both stabilizable and detectable via the controls and measurements chosen (see discussion at the end of this section), this results in finite values for $\boldsymbol{u}, \boldsymbol{v}$ and $\boldsymbol{w}$, the magnitudes of which may be adjusted by variation of the three scalar parameters $\ell, \alpha$ and $\gamma$, respectively. Reducing $\ell$ (which scales the control penalty in the cost function) generally results in increased control feedback $\boldsymbol{u}^{*} \boldsymbol{R} \boldsymbol{u}$ and reducing $\alpha$ (which scales the measurement noise in the governing equation) generally results in increased estimator feedback $\boldsymbol{v}^{*} \boldsymbol{v}$. Reducing $\gamma$ (which scales the disturbance penalty in the cost function) generally results in increased disturbances $\boldsymbol{w}^{*} \boldsymbol{S} w$ with the maximally malevolent structure when designing the most suitable feedback rules for $\boldsymbol{u}$ and $\boldsymbol{v}$ to compensate, in the spirit of a non-cooperative game; the $\mathscr{H}_{\infty}$ approach is thus often referred to as a robust control approach. The $\mathscr{H}_{\infty}$ control solution first derived in Doyle et al. (1989) may be described as a saddle-point solution as follows: a compensator which minimizes $\mathscr{J}$ in the presence of that disturbance which simultaneously maximizes $\mathscr{J}$ is given by

$$
\boldsymbol{K}=-\frac{1}{\ell^{2}} \boldsymbol{R}^{-1} \boldsymbol{B}^{*} \boldsymbol{X}, \quad \boldsymbol{L}=-\frac{1}{\alpha^{2}} \mathbf{Z} \mathbf{Y} \boldsymbol{C}^{*} \mathbf{G}_{2}^{*-1} \boldsymbol{S}_{2} \mathbf{G}_{2}^{-1}
$$

with

$$
\hat{\boldsymbol{w}}_{1}=\frac{1}{\gamma^{2}} \boldsymbol{S}_{1}^{-1} \boldsymbol{G}_{1}^{*} \boldsymbol{X} \hat{\boldsymbol{x}}, \quad \hat{\boldsymbol{w}}_{2}=0
$$


and where

$$
\left.\begin{array}{l}
\boldsymbol{X}=\operatorname{Ric}\left(\begin{array}{cc}
\boldsymbol{A} & \frac{1}{\gamma^{2}} \boldsymbol{G}_{1} \boldsymbol{S}_{1}^{-1} \boldsymbol{G}_{1}^{*}-\frac{1}{\ell^{2}} \boldsymbol{B} \boldsymbol{R}^{-1} \boldsymbol{B}^{*} \\
-\boldsymbol{Q} & -\boldsymbol{A}^{*}
\end{array}\right), \\
\boldsymbol{Y}=\operatorname{Ric}\left(\begin{array}{cc}
\boldsymbol{A}^{*} & \frac{1}{\gamma^{2}} \boldsymbol{Q}-\frac{1}{\alpha^{2}} \boldsymbol{C}^{*} \boldsymbol{G}_{2}^{*-1} \boldsymbol{S}_{2} \boldsymbol{G}_{2}{ }^{-1} \boldsymbol{C} \\
-\boldsymbol{G}_{1} \boldsymbol{S}_{1}^{-1} \boldsymbol{G}_{1}^{*} & -\boldsymbol{A}
\end{array}\right), \\
\mathbf{Z}=\left(I-\frac{\boldsymbol{Y} \boldsymbol{X}}{\gamma^{2}}\right)^{-1} .
\end{array}\right\}
$$

Note that

$$
\operatorname{Ric}\left(\begin{array}{cc}
\boldsymbol{A} & -\boldsymbol{B} \\
-\boldsymbol{C} & -\boldsymbol{A}^{*}
\end{array}\right)
$$

denotes the unique positive-definite solution $\boldsymbol{X}$ of the associated algebraic Riccati equation $0=\boldsymbol{X} \boldsymbol{A}+\boldsymbol{A}^{*} \boldsymbol{X}-\boldsymbol{X} \boldsymbol{B} \boldsymbol{X}+\boldsymbol{C}$. A lower bound on $\gamma$ for which the control problem may be solved, $\gamma_{0}$, may be found by trial and error. The $\gamma \rightarrow \infty$ limit of this approach is referred to as $\mathscr{H}_{2}$ or linear quadratic Gaussian (LQG) control, and is also referred to simply as optimal control.

Three conditions are necessary and sufficient for the $\mathscr{H}_{\infty}$ control solution to exist: (i) the system matrix $\boldsymbol{A}$ has to be stabilizable by the input matrix $\boldsymbol{B}$, meaning that it is possible to stabilize all the unstable modes of $\boldsymbol{A}$ with an appropriate input, (ii) the matrix $\boldsymbol{A}$ has to be detectable by the observation matrix $\boldsymbol{C}$, meaning that it is possible for the output to measure the dynamics of all the unstable modes of $\boldsymbol{A}$, and (iii) a coupling condition $\rho(\boldsymbol{X Y})<\gamma^{2}$, where $\rho(M)$ denotes the maximum eigenvalue of $M$, has to be satisfied by two positive-definite matrices $\boldsymbol{X}$ and $\boldsymbol{Y}$ which solve the above Riccati equations. When $\gamma=\infty$, the third requirement vanishes and the first two are, in fact, necessary and sufficient for the solution, which is unique in this case, to exist. When $\gamma<\infty$, even if the first two requirements are satisfied, if $\gamma$ is too small, positive-definite solutions $\boldsymbol{X}$ and $\boldsymbol{Y}$ to the above Riccati equations might not exist, or the coupling condition $\rho(\boldsymbol{X Y})<\gamma^{2}$ might be violated even if such solutions $\boldsymbol{X}$ and $\boldsymbol{Y}$ do exist. In other words, in the case with $\gamma<\infty$, stabilizability and detectability of the system are not sufficient for the $\mathscr{H}_{\infty}$ control problem to be solvable.

Further, when a solution to the $\mathscr{H}_{\infty}$ control problem in the case $\gamma_{0}<\gamma<\infty$ does exist, it is not unique; alternative solutions might be of particular interest in secondary minimization problems which are not addressed here. Note that both the $\mathscr{H}_{2}$ and $\mathscr{H}_{\infty}$ problems, as formulated above, involve solving two Riccati equations and thus require almost the same computational effort. In the present work, the Riccati equations were solved using both the Schur decomposition approach (Laub 1991) and the standard eigenvalue decomposition approach (Zhou \& Doyle 1997) using double-precision arithmetic. As discussed in detail in Lauga \& Bewley (2003), particular care is needed in the numerical solution of these Riccati equations as the Reynolds number is increased, as the corresponding Riccati equations become increasingly difficult to solve and double-precision arithmetic (the default precision in Matlab) becomes inadequate above $R e \gtrsim 200$. The present work avoids these difficulties by focusing its attention on cases with $R e<190$.

The simple structure of the $\mathscr{H}_{\infty}$ control solution, and its profound implications in terms of the performance and robustness of the resulting closed-loop system, is one of the most elegant results of linear control theory, and is discussed in greater detail 
in, e.g. Doyle et al. (1989) and Green \& Limebeer (1995), and in a fluid-mechanical setting in Bewley \& Liu (1998). (Note that Bewley \& Liu (1998) omitted the term $\hat{\boldsymbol{w}}$ and assumed $\mathbf{Z}=\boldsymbol{I}$ in the compensator formulation. While the formulation listed there was correct in the $\mathscr{H}_{2}$ limit (for $\gamma \rightarrow \infty$ ), including the $\mathbf{Z}$ and $\hat{\boldsymbol{w}}$ terms correctly, as done here, might significantly improve the performance of the $\mathscr{H}_{\infty}$ compensator.) It is useful to state an alternative interpretation of the $\mathscr{H}_{\infty}$ control solution, as presented in the lucid texbook by Green \& Limebeer (1995). Instead of interpreting the solution in a non-cooperative framework, an alternative interpretation can be given in terms of operator norms, where the control $\boldsymbol{u}$ minimizes the cost functional

$$
\tilde{\mathscr{J}}=\mathscr{E}\left[\boldsymbol{x}^{*} \boldsymbol{Q} \boldsymbol{x}+\ell^{2} \boldsymbol{u}^{*} \boldsymbol{R} \boldsymbol{u}\right]
$$

under the noise-amplification constraint $\tilde{\mathscr{J}} \leqslant \gamma^{2} \mathscr{E}\left[\boldsymbol{w}^{*} \boldsymbol{S} \boldsymbol{w}\right]$. The control solution is the same as the one given above, while making clear the rationale for applying $\mathscr{H}_{\infty}$ control instead of $\mathscr{H}_{2}$ control: the $\mathscr{H}_{\infty}$ control design prescribes an upper bound on the noise amplification by the closed-loop system. Reducing this upper bound from infinity (its $\mathscr{H}_{2}$ value) can sometimes facilitate a significant decrease in the system sensitivity to external disturbances of a particular disruptive structure.

\subsection{Techniques used to characterize closed-loop system performance}

\subsubsection{Linear stabilizability and detectability}

The first objective of the control design is simply to stabilize the closed-loop linear system. In the $\mathscr{H}_{2}$ setting described above, this is possible when the linear system considered is both stabilizable and detectable. As discussed in detail in Lauga \& Bewley (2003), the present linear system is, in theory, stabilizable at any Reynolds number; it follows via a very similar analysis that the linear system is also, in theory, detectable at any Reynolds number. Thus, using sufficiently high-precision arithmetic and a sufficiently high value of $\gamma$ in the $\mathscr{H}_{\infty}$ control design, linearly stabilizing control feedback may, in theory, be found for the present system at any Reynolds number, though the performance of the linear control designs at high Reynolds numbers is so bad that, in practice, they most assuredly will fail to stabilize the full nonlinear system. The difficulty is that as the Reynolds number is increased and an increasing number of modes become open-loop unstable, the controllability and observability of these open-loop unstable modes, though not zero, become exponentially small (Lauga \& Bewley 2003). Thus, the problem of characterizing the controlled linear system is significantly more delicate than just the binary characterizations of linear stabilization and detection. To characterize the linear and nonlinear performance of the controlled system, other metrics must be used, as described below.

\subsubsection{Linear transient energy growth}

As mentioned in the previous paragraph, stabilization of the closed-loop eigenvalues of the system (that is, moving the closed-loop system eigenvalues into the left halfplane) only partially characterizes the behaviour of the controlled linear system. As shown in Lauga \& Bewley (2003), the diminishing controllability and observability of the open-loop unstable modes as the Reynolds number is increased, as discussed above, manifests itself by the increasing non-normality of the closed-loop system eigenvectors as the Reynolds number is increased to the point that, at high Reynolds numbers, the closed-loop linear system is nearly defective. Both the transient response of the unforced closed-loop linear system from perturbed initial conditions and the statistics of the stochastically forced closed-loop linear system are strong functions of the degree of non-normality of the closed-loop system eigenvectors. In particular, 
initial conditions formed by destructive linear combinations of the non-normal system eigenvectors can sometimes lead to very large transient energy growth in a stable system; thus, very small initial perturbations can sometimes be efficiently amplified by such linear mechanisms and lead to nonlinear instability of the full system even though it is linearly stable (for an example of how this happens commonly in uncontrolled transitional flows, see, e.g. Schmid \& Henningson 2001). Hence, nonnormality of the closed-loop system eigenvectors has important implications for the application of linear control to the nonlinear system. We will quantify this effect in this paper by computing the worst possible energy amplification by the closed-loop linear system,

$$
E_{\max }=\left[\max _{\boldsymbol{x}(0), \tau} \frac{\boldsymbol{x}^{*}(\tau) \boldsymbol{Q} \boldsymbol{x}(\tau)}{\boldsymbol{x}^{*}(0) \boldsymbol{Q} \boldsymbol{x}(0)}\right]^{1 / 2},
$$

over all possible initial conditions $\boldsymbol{x}(0)$ and finite time horizons $\tau$. $E_{\max }$ is computed using a standard matrix norm method, as detailed in Schmid \& Henningson (2001).

\subsubsection{Transfer function norms}

Another important goal of the control design is disturbance rejection, that is, keeping the response of the system to external disturbances as small as possible. This objective may be quantified by computing input-output transfer function norms $\left\|\boldsymbol{T}_{\tilde{z} \tilde{w}}\right\|$ in the closed-loop setting. As discussed further in Bewley \& Liu (1998), the analysis can be made on a generic closed-loop system (i.e. one for which a control design has already closed the control and/or estimation loops) written in the standard state-space form:

$$
\left.\begin{array}{l}
\dot{\tilde{\boldsymbol{x}}}=\tilde{\boldsymbol{A}} \tilde{\boldsymbol{x}}+\tilde{\boldsymbol{B}} \tilde{\boldsymbol{w}} \\
\tilde{z}=\tilde{\mathbf{C}} \tilde{\boldsymbol{x}} .
\end{array}\right\}
$$

The output of interest $\tilde{z}$ in this generic form can, for example, be some scaling of the state $\boldsymbol{x}$, the control $\boldsymbol{u}$, or a linear combination of the two. Two specific quantities of interest are the transfer function 2-norm and the transfer function $\infty$-norm. Loosely speaking, these norms quantify how the wake model with control applied responds to 'benign' and 'malevolent' disturbances, respectively.

The transfer function two-norm $\left\|\boldsymbol{T}_{\tilde{z} \tilde{w}}\right\|_{2}$ is defined as the expected value of the mean square of system output $\tilde{z}$ when the system (3.7) is driven by white Gaussian disturbances $\tilde{\boldsymbol{w}}$ of prescribed variance, that is, assuming some disturbance covariance structure $\mathscr{E}\left[\tilde{\boldsymbol{w}} \tilde{\boldsymbol{w}}^{*}\right]=\tilde{\boldsymbol{s}}^{-1}$,

$$
\left\|\boldsymbol{T}_{\tilde{z} \tilde{z}}\right\|_{2}^{2}=\mathscr{E}\left[\tilde{z}^{*} \tilde{z}\right] .
$$

In particular, by coordinating the control $\boldsymbol{u}$ with the measurements $\boldsymbol{y}$ in the system (2.7) with a dynamic compensator, the $\mathscr{H}_{2}$ control design is constructed to exactly minimize

$$
\mathscr{J}=\mathscr{E}\left[z^{*} z\right] \quad \text { where } \quad z=\left(\begin{array}{c}
\mathbf{Q}^{1 / 2} \\
0
\end{array}\right) \boldsymbol{x}+\ell\left(\begin{array}{c}
0 \\
\boldsymbol{R}^{1 / 2}
\end{array}\right) \boldsymbol{u},
$$

in the presence of white Gaussian disturbances $\boldsymbol{w}$ with covariance $\mathscr{E}\left[\boldsymbol{w} \boldsymbol{w}^{*}\right]=\boldsymbol{S}^{-1}$. Thus, it follows that the $\mathscr{H}_{2}$ control design minimizes

$$
\left\|\boldsymbol{T}_{z w}\right\|_{2}^{2}=\left\|\boldsymbol{T}_{x w}\right\|_{2}^{2}+\ell^{2}\left\|\boldsymbol{T}_{u w}\right\|_{2}^{2},
$$

where $\left\|\boldsymbol{T}_{\boldsymbol{x}}\right\|_{2}$ and $\left\|\boldsymbol{T}_{\boldsymbol{u} w}\right\|_{2}$ represent, respectively, the two-norms of the transfer functions from disturbances $\boldsymbol{w}$ (of covariance $\boldsymbol{S}^{-1}$ ) to the state $\boldsymbol{x}$ (weighted by $\mathbf{Q}^{1 / 2}$ ) and to the control $\boldsymbol{u}$ (weighted by $\boldsymbol{R}^{1 / 2}$ ). The latter can usually be interpreted as a measure of the control energy used by the control design in the presence of Gaussian 
disturbances. The two-norms may be computed using a trace formula involving either the controllability Gramian $\tilde{L}_{c}$ of the realization $(\tilde{\boldsymbol{A}}, \tilde{\boldsymbol{B}})$ or the observability $\tilde{L}_{o}$ Gramian of the realization $(\tilde{\boldsymbol{A}}, \tilde{\boldsymbol{C}})$ (Green \& Limebeer 1995).

The $\mathscr{H}_{2}$ control design minimizes the expected (that is, time-average) amplification of disturbances of Gaussian structure by the system over the class of all stabilizing controllers. In many problems, however, we can argue that instead of considering as an input a particular stochastic process (namely, Gaussian disturbances), a more relevant quantity to compute is the maximum amplification possible over all bounded disturbances. This quantity is the second performance index we will use, and is called the transfer function infinity norm. In the generic setting of the system (3.7), it is defined as the operator norm

$$
\left\|\boldsymbol{T}_{\tilde{z} \tilde{\boldsymbol{w}}}\right\|_{\infty}^{2}=\max _{\tilde{\boldsymbol{w}}} \frac{\mathscr{E}\left[\tilde{z}^{*} \tilde{z}\right]}{\mathscr{E}\left[\tilde{\boldsymbol{w}}^{*} \tilde{\boldsymbol{S}} \tilde{\boldsymbol{w}}\right]},
$$

and may be computed iteratively (Doyle et al. 1989). The value of $\tilde{\boldsymbol{w}}$ that achieves the maximum in this expression is the worst-case disturbance which adjusts continuously in time to produce the largest energy amplification in the system on the infinite horizon. Note in particular that $\mathscr{H}_{\infty}$ control minimizes $\left\|\boldsymbol{T}_{z w}\right\|_{2}$ while bounding $\left\|\boldsymbol{T}_{z w}\right\|_{\infty}<\gamma$. The transfer function infinity norms presented in this paper have been determined with a $0.5 \%$ tolerance of error.

\section{Control of the linear wake model}

The linear control strategy outlined in the previous section was tested over a range of relevant parameters in the control formulation. We now quantify the performance of this control strategy on the linear CGL system using two values for the control penalty in the cost function $(\ell=1000$, which results in relatively weak control feedback, and $\ell=0.1$, which results in relatively strong control feedback) and two values for the measurement-noise-to-state-disturbance ratio $(\alpha=1000$, which implies a large amount of measurement noise and results in relatively weak corrections to the estimator with feedback based on the measurements, and $\alpha=0.1$, which implies little measurement noise and results in relatively strong corrections to the estimator with feedback based on the measurements). Examination of the linear control effectiveness on the full nonlinear system is deferred to $\S 5$.

\subsection{Examination of eigenvalues: root loci}

As mentioned previously, stabilization of the closed-loop eigenvalues of the system (that is, moving the closed-loop eigenvalues into the left half-plane) partially characterizes the effect of control feedback on the system. A root locus plot characterizes the movement of the closed-loop system eigenvalues as a scalar parameter in the control formulation is varied. In classical control approaches, this scalar control design parameter is almost always selected to be the overall feedback gain $K$. In our present modern control framework, there are three scalar control design parameters of interest: $\{\ell, \alpha, \gamma\}$. Two root loci of the controlled system in the state feedback setting (that is, based on full state information) are illustrated here; the root loci characterizing (partially) the closed-loop dynamics of the state estimation error demonstrate similar trends.

Figure 2 displays a 'root locus with respect to $\ell$ ', that is, the locus of the first several eigenvalues of the wake model at $R e=175$ before $(\square)$ and after state-feedback control is applied for control penalties ranging continuously from $\ell=1000(\diamond)$ to $\ell=0.01$ 


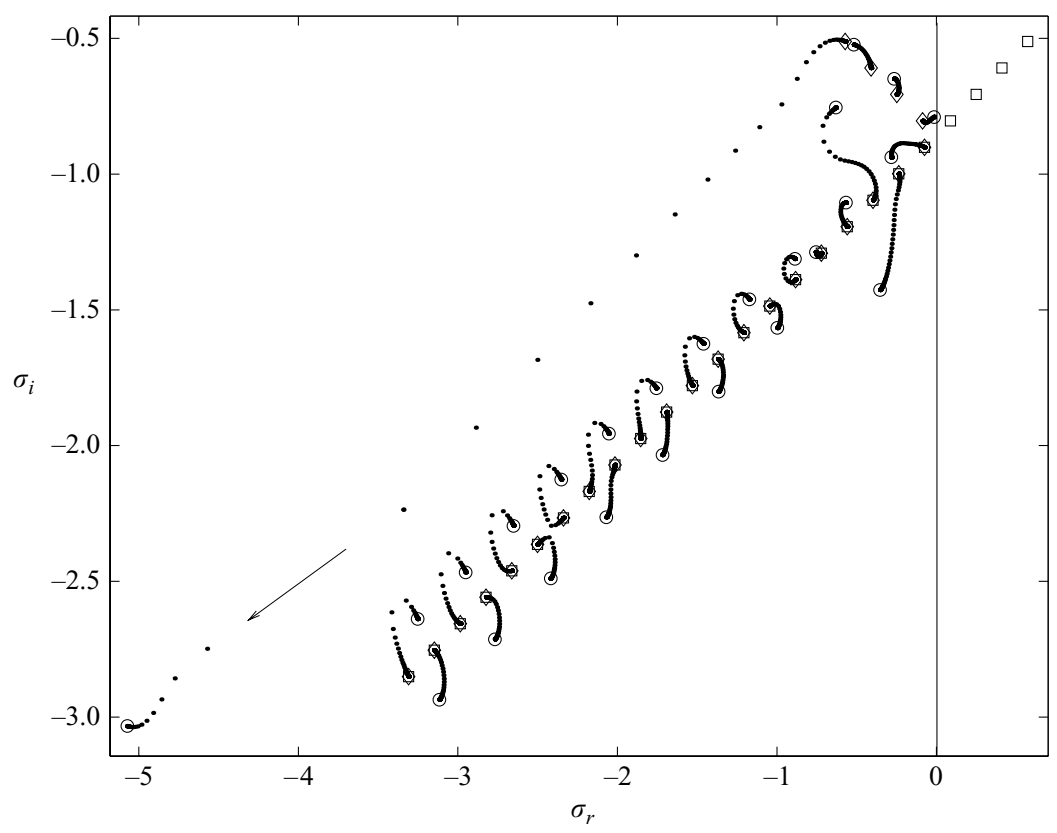

FIGURE 2. Comparison of the eigenvalues of the linear closed-loop wake model at $R e=175$ without control ( $\square$ ) and with full-information $\mathscr{H}_{2}$ control applied as a function of the control penalty $\ell$, from $\ell=1000(\diamond)$ to $\ell=0.01(\bigcirc)$. This plot is referred to as 'root locus with respect to $\ell$ '.

$(\bigcirc)$, taking $\gamma=\infty$. The first observation to make in this figure is that the minimumenergy control (with $\ell=1000$ ) does not simply move the unstable eigenvalues of the model just to the stable side of the imaginary axis, but actually reflects them across the imaginary axis into the stable half-plane (this phenomenon is well known in the field of control theory). As the strength of the control feedback is allowed to increase (by decreasing $\ell$ in the cost function), the eigenvalues move in a non-trivial manner, some shifting to the left and some shifting to the right, with some moving a great deal and some moving only a little.

Figure 3 displays a 'root locus with respect to $\gamma$ ', that is, the locus of the first several eigenvalues of the wake model at $R e=175$ before ( $\square$ ) and after state-feedback control is applied, for robustness parameters ranging continuously from $\gamma=\infty(\diamond)$ to $\gamma=\gamma_{0}$ $(\bigcirc)$, taking $\ell=1000$. The striking feature displayed by figure 3 is that the effect of decreasing $\gamma$ is much more targeted, moving only a few of the eigenvalues (specifically, in the present case, those corresponding to the open-loop unstable linear modes).

While the trends in such plots look interesting, root locus plots fail to provide a complete picture of the effect of control feedback on a highly non-normal system such as the present. This is because, as mentioned previously, the eigenvectors are significantly modified by the control feedback, and this has a very important effect on the closed-loop system dynamics. This information is not evident in a root locus plot.

\subsection{Comparison of performance: modern vs. proportional control}

The proportional measurement feedback scheme proposed and tested in RM96 may, in the present notation, be written as

$$
u(x, t)=|g| \exp \left(\mathrm{i} \phi_{g}\right) \delta\left(x-x_{f}\right) \psi\left(x_{s}\right) \quad \Rightarrow \quad \boldsymbol{u}=|g| \exp \left(\mathrm{i} \phi_{g}\right) \boldsymbol{y} .
$$




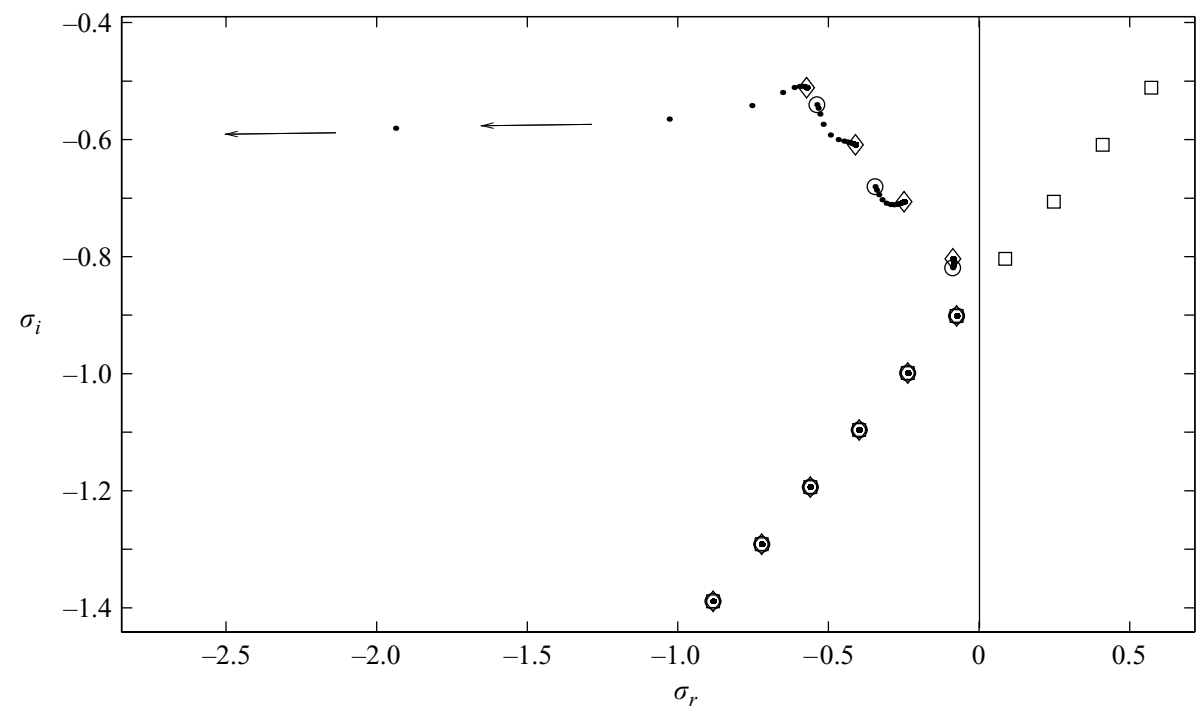

FIGURE 3. Comparison of the eigenvalues of the linear closed-loop wake model at $R e=175$ without control $(\square)$ and with full information $\mathscr{H}_{\infty}$ control applied as a function of the robustness parameter $\gamma$, from $\gamma=\infty(\diamond)$ to $\gamma=\gamma_{0}(\bigcirc)$. The control penalty is $\ell=1000$. This plot is referred to as 'root locus with respect to $\gamma$ '.

There are two degrees of freedom in this scheme, the phase $\phi_{g}$ and the amplitude $|g|$. Effective values of these parameters (RM96) are $\phi_{g}=0.42 \pi$ and $|g|=0.0048\left(R e-R e_{c}\right)$. This existing proportional control strategy for the present system will be used as a point of comparison for the present study. The RM96 control scheme was capable of stabilizing only one open-loop unstable global mode in RM96 (that is, it could stabilize the linear system up to $R e=64$ ); our own numerical tests verified this. On the other hand, as discussed in Lauga \& Bewley (2003), the present modern control design is able to linearly stabilize several open-loop unstable global modes with a single sensor/actuator pair. To be specific, using double-precision arithmetic, six openloop unstable global modes may be linearly stabilized (that is, the present control design could stabilize the linear system up to $R e=284$ ); this number is significantly smaller if single-precision arithmetic is used and significantly larger if quad-precision arithmetic is used. Another advantage of the modern control design over simple proportional feedback is a significant reduction in both transient energy growth and input/output transfer function norms. Table 1 presents a comparison between the values of the transfer function 2-norms $\left\|\boldsymbol{T}_{x w}\right\|_{2},\left\|\boldsymbol{T}_{\boldsymbol{u}}\right\|_{2}$, the transfer function infinitynorm $\left\|\boldsymbol{T}_{x w}\right\|_{\infty}$, and the maximum transient energy growth $E_{\max }$ using the proportional, $\mathscr{H}_{2}$, and $\mathscr{H}_{\infty}\left(\gamma_{0}\right)$ control design approaches.

As seen in table 1 , the $\mathscr{H}_{2}$ and $\mathscr{H}_{\infty}\left(\gamma_{0}\right)$ control designs generally result in smaller values for both the transient energy growth $E_{\max }$ and the transfer function norms $\left\|\boldsymbol{T}_{x w}\right\|_{2}$ and $\left\|\boldsymbol{T}_{x w}\right\|_{\infty}$ than the proportional control design of RM96. This illustrates that the modern control approach typically makes the closed-loop system less prone to amplify perturbations in initial conditions and less sensitive to external disturbances of both Gaussian and worst-case structure. Both results indicate that the closed-loop system is made closer to normal (that is, lessening the severity of the non-normality of the closed-loop system eigenvectors) by the modern control design than by the proportional control design. We also see that, for large $\ell$, the $\mathscr{H}_{2}$ control designs 


\begin{tabular}{|c|c|c|c|c|c|c|}
\hline & Control & & $\left\|\boldsymbol{T}_{\boldsymbol{x} w}\right\|_{2}$ & $\left\|\boldsymbol{T}_{u w}\right\|_{2}$ & $\left\|\boldsymbol{T}_{x w}\right\|_{\infty}$ & $E_{\max }$ \\
\hline$\alpha=1000$ & $\begin{array}{l}\text { RM96 } \\
\mathscr{H}_{2}(\gamma=\infty) \\
\mathscr{H}_{\infty}\left(\gamma=\gamma_{0}\right)\end{array}$ & $\begin{array}{l}-\bar{\ell}=1000 \\
\ell=0.1 \\
\ell=1000 \\
\ell=0.1\end{array}$ & $\begin{array}{r}2050 \\
1610 \\
954 \\
1060 \\
2520\end{array}$ & $\begin{array}{c}58.6 \\
58.3 \\
660 \\
2280 \\
9090\end{array}$ & $\begin{array}{r}24200 \\
13400 \\
5590 \\
6820 \\
2890\end{array}$ & $\begin{array}{l}7.35 \\
6.03\end{array}$ \\
\hline$\alpha=0.1$ & $\begin{array}{l}\text { RM96 } \\
\mathscr{H}_{2}(\gamma=\infty) \\
\mathscr{H}_{\infty}\left(\gamma=\gamma_{0}\right)\end{array}$ & $\begin{array}{l}\bar{\ell}=1000 \\
\ell=0.1 \\
\ell=1000 \\
\ell=0.1\end{array}$ & $\begin{array}{l}55.9 \\
21.1 \\
7.65 \\
8.75 \\
7.77\end{array}$ & $\begin{array}{r}1.60 \\
0.93 \\
7.72 \\
8.83 \\
10.7\end{array}$ & $\begin{array}{c}654 \\
131 \\
15.4 \\
31.9 \\
13.0\end{array}$ & 2 \\
\hline
\end{tabular}

TABLE 1. Comparison of the transfer function 2-norms $\left\|\boldsymbol{T}_{x \boldsymbol{w}}\right\|_{2}$ and $\left\|\boldsymbol{T}_{\boldsymbol{u}}\right\|_{2}$, the transfer function $\infty$-norm $\left\|\boldsymbol{T}_{x w}\right\|_{\infty}$, and the maximum transient energy growth $E_{\max }$, using the proportional control strategy of RM96 and the modern $\mathscr{H}_{2}(\gamma=\infty)$ and $\mathscr{H}_{\infty}\left(\gamma=\gamma_{0}\right)$ control strategies in the linear wake model at $R e=60$, taking two values of the measurement-noise-to-state-disturbance ration $\alpha$.

$\begin{array}{clcccc}\text { Re } & & \mathscr{H}_{2} & \text { control } & & \\ 100 & \ell=1000 & 21.5 & 2.89 & 126 & 4.37 \\ & \ell=0.1 & 10.6 & 12.0 & 27.3 & 4.73 \\ 150 & \ell=1000 & 412 & 65.0 & 351 & 82.6 \\ & \ell=0.1 & 158 & 233 & 489 & 49.2 \\ & & \boldsymbol{T}_{\boldsymbol{x} w} \|_{2} & \left\|\boldsymbol{T}_{\boldsymbol{u w}}\right\|_{2} & \left\|\boldsymbol{T}_{\boldsymbol{x} w}\right\|_{\infty} & E_{\text {max }} \\ \operatorname{Re} & & \| \boldsymbol{T}_{\boldsymbol{x}}\left(\gamma_{2}\right) & \text { control } & & \\ 100 & \ell=1000 & 12.4 & 13.2 & 53.1 & 2.97 \\ & \ell=0.1 & 14.3 & 43.0 & 16.9 & 15.0 \\ 150 & \ell=1000 & 254 & 283 & 2080 & 52.9 \\ & \ell=0.1 & 195 & 542 & 293 & 81.5\end{array}$

TABLE 2. Transfer function 2-norms $\left\|\boldsymbol{T}_{x w}\right\|_{2}$ and $\left\|\boldsymbol{T}_{\boldsymbol{u}}\right\|_{2}$, $\infty$-norm $\left\|\boldsymbol{T}_{x \boldsymbol{w}}\right\|_{\infty}$ and maximum transient energy growth $E_{\max }$ of the linear wake model with full information $\mathscr{H}_{2}$ (top) and $\mathscr{H}_{\infty}\left(\gamma=\gamma_{0}\right.$, bottom) control applied as a function of the Reynolds number for two values of $\ell$.

outperform the proportional control design in terms of both the disturbance-to-state transfer function norm, $\left\|\boldsymbol{T}_{x w}\right\|_{2}$, and the disturbance-to-control transfer function norm, $\left\|\boldsymbol{T}_{u w}\right\|_{2}$. However, the control energy required by both the $\mathscr{H}_{\infty}\left(\gamma_{0}\right)$ designs and the $\mathscr{H}_{2}(\ell=0.1)$ designs are large as compared with the other approaches. Generally, a trade-off must be made between the various metrics of interest during the control design process, and the norms $\left\|\boldsymbol{T}_{x w}\right\|_{2}$ and $\left\|\boldsymbol{T}_{x w}\right\|_{\infty}$ may be reduced at the price of increasing $\left\|\boldsymbol{T}_{u w}\right\|_{2}$ by adjusing $\ell$ and $\gamma$. In most cases, intermediate values of both $\ell$ and $\gamma$ would be preferred.

\subsection{Comparison of performance: $\mathscr{H}_{2}$ vs. $\mathscr{H}_{\infty}$ control designs}

We now compare the performances of the $\mathscr{H}_{2}$ and $\mathscr{H}_{\infty}\left(\gamma_{0}\right)$ control strategies for both the full-information and the estimator-based configurations at $R e=100$ and 150 . Note the proportional control strategy discussed earlier fails to stabilize the linear CGL system modelling the cylinder wake at Reynolds numbers this high. Table 2 displays the transfer function norms $\left\|\boldsymbol{T}_{x w}\right\|_{2},\left\|\boldsymbol{T}_{u w}\right\|_{2}$ and $\left\|\boldsymbol{T}_{x w}\right\|_{\infty}$, and the maximum 


\begin{tabular}{|c|c|c|c|c|c|c|}
\hline$R e$ & & & $\left\|\boldsymbol{T}_{x w}\right\|_{2}^{\mathscr{H}_{2}}$ & $\begin{array}{l}\text { control } \\
\qquad \boldsymbol{T}_{\boldsymbol{u w}} \|_{2}\end{array}$ & $\left\|\boldsymbol{T}_{x w}\right\|_{\infty}$ & $E_{\max }$ \\
\hline \multirow[t]{2}{*}{100} & $\alpha=1000$ & $\begin{array}{l}\ell=1000 \\
\ell=0.1\end{array}$ & $\begin{array}{r}21500 \\
8910\end{array}$ & $\begin{array}{r}2930 \\
12300\end{array}$ & $\begin{array}{r}144000 \\
36200\end{array}$ & $\begin{array}{c}34.2 \\
8.70\end{array}$ \\
\hline & $\alpha=0.1$ & $\begin{array}{l}\ell=1000 \\
\ell=0.1\end{array}$ & $\begin{array}{l}75.4 \\
35.6\end{array}$ & $\begin{array}{l}10.2 \\
41.1\end{array}$ & $\begin{array}{l}538 \\
191\end{array}$ & $\begin{array}{l}11.9 \\
13.7\end{array}$ \\
\hline \multirow[t]{4}{*}{150} & $\alpha=1000$ & $\ell=1000$ & 3660000 & 549000 & 31700000 & 2920 \\
\hline & & $\ell=0.1$ & 1280000 & 2030000 & 351000 & 1350 \\
\hline & $\alpha=0.1$ & $\ell=1000$ & 12200 & 1830 & 106000 & 1640 \\
\hline & & $\ell=0.1$ & 4400 & 6840 & 12400 & 1190 \\
\hline$R e$ & & & $\begin{array}{l}\mathscr{H}_{\infty}\left(\gamma_{0}\right) \\
\left\|\boldsymbol{T}_{\boldsymbol{x}}\right\|_{2}\end{array}$ & $\begin{array}{l}\text { control } \\
\qquad \boldsymbol{T}_{u w} \|_{2}\end{array}$ & $\left\|\boldsymbol{T}_{x w}\right\|_{\infty}$ & $E_{\max }$ \\
\hline \multirow[t]{4}{*}{100} & $\alpha=1000$ & $\ell=1000$ & 12000 & 1600 & 68900 & 25.5 \\
\hline & & & 15600 & 569 & 18000 & 23.1 \\
\hline & $\alpha=0.1$ & $\ell=1000$ & 43.9 & 10 & 252 & 67.8 \\
\hline & & $\ell=$ & 73.2 & 233 & 96.8 & 82.0 \\
\hline \multirow[t]{4}{*}{150} & $\alpha=1000$ & $\ell=1000$ & 2130000 & 3700000 & 17600000 & 5400 \\
\hline & & $\ell=0.1$ & 1740000 & 6400000 & 1980000 & 864 \\
\hline & $\alpha=0.1$ & $\ell=1000$ & 7340 & 10300 & 58300 & 1740 \\
\hline & & $\ell=0.1$ & 6240 & 23500 & 6920 & 3590 \\
\hline
\end{tabular}

TABLE 3. Transfer function 2-norms $\left\|\boldsymbol{T}_{x w}\right\|_{2}$ and $\left\|\boldsymbol{T}_{\boldsymbol{u}}\right\|_{2}$, $\infty$-norm $\left\|\boldsymbol{T}_{\boldsymbol{x} w}\right\|_{\infty}$ and maximum transient energy growth $E_{\text {max }}$ of the linear wake model with estimator-based $\mathscr{H}_{2}$ (top) and $\mathscr{H}_{\infty}\left(\gamma=\gamma_{0}\right.$, bottom $)$ control applied as a function of the Reynolds number for two values of $\ell$ and two values of $\alpha$.

transient energy growth $E_{\text {max }}$, for two controls strategies: the full-information $\mathscr{H}_{2}$ control and the full information $\mathscr{H}_{\infty}$ control with the smallest value possible for $\gamma$. Table 3 repeats these calculations in the case where a dynamic compensator is used to compute the control based on noisy measurements at the point sensor location $x_{s}$.

\subsubsection{General characteristics}

Most of the trends depicted in tables 2 and 3 are what we might expect before actually calculating the numbers. For example, $\left\|\boldsymbol{T}_{z w}\right\|_{2}$ is always smaller than $\left\|\boldsymbol{T}_{z w}\right\|_{\infty}$, since the former quantifies the amplification by the system of disturbances of a particular, somewhat 'benign' structure (zero-mean, uncorrelated, white Gaussian), whereas the latter quantifies worst-case amplification by the system of any bounded disturbances of any structure. Thus, recalling the relations between $z$ and $\boldsymbol{x}$ in (3.8) and (3.9), it is often found (see tables 2 and 3) that $\left\|\boldsymbol{T}_{x w}\right\|_{2}$ is also smaller than $\left\|\boldsymbol{T}_{x w}\right\|_{\infty}$, though this relation does not hold in general.

For a given Reynolds number, when $\mathscr{H}_{2}$ control is applied, the transfer function norms depend monotonically on the control penalty $\ell$ and the measurement-noise-tostate-disturbance ratio $\alpha$. For a given $\alpha$, reducing $\ell$ results in the application of more control (a larger value for $\left\|\boldsymbol{T}_{u w}\right\|_{2}$ ) and an improved disturbance rejection (smaller values for the transfer function norms $\left\|\boldsymbol{T}_{x w}\right\|_{2}$ and $\left.\left\|\boldsymbol{T}_{x w}\right\|_{\infty}\right)$. For a given $\ell$, increasing $\alpha$ corresponds to having less reliable measurements, resulting in more control effort to achieve stabilization (larger $\left\|\boldsymbol{T}_{u w}\right\|_{2}$ ) and a degradation in the disturbance rejection (larger values for the transfer function norms $\left\|\boldsymbol{T}_{x w}\right\|_{2}$ and $\left\|\boldsymbol{T}_{x w}\right\|_{\infty}$ ).

Such observations do not extend directly to the variations of the maximum transient energy growth with $\ell$ and $\alpha$. For example, for a given $\alpha$ in table $3, E_{\max }$ sometimes 

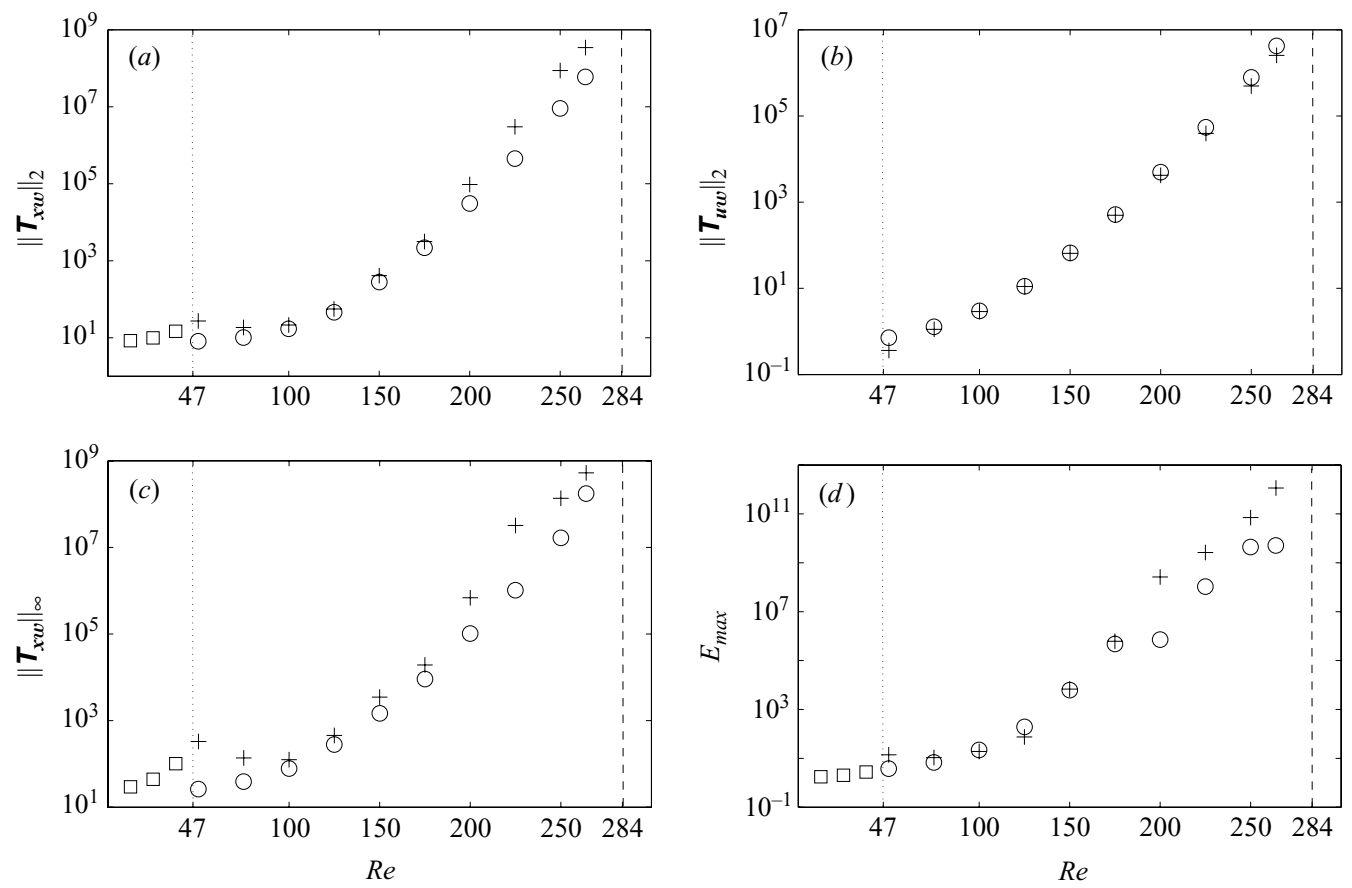

FIGURE 4. Performance of the uncontrolled $(\square, R e<47)$ and controlled linear wake model as a function of Reynolds number for two control penalties $(+) \ell=1000,(\bigcirc) \ell=0.1$ using full information $\mathscr{H}_{2}$ control. (a) Transfer function 2-norm $\left\|\boldsymbol{T}_{\boldsymbol{x}}\right\|_{2} ;(b)$ transfer function 2-norm $\left\|\boldsymbol{T}_{\boldsymbol{u}}\right\|_{2} ;(c)$ transfer function $\infty$-norm $\left\|\boldsymbol{T}_{\boldsymbol{x} w}\right\|_{\infty} ;(d)$ maximum transient energy growth $E_{\text {max }}$. All plots are semilog- $y$ and were computed using double precision arithmetic; note that, at this precision, stabilizing linear control feedback could not be determined above $R e \approx$ 284.

increases with $\ell$ and sometimes decreases with $\ell$. Such behaviour is to be expected, as the $\mathscr{H}_{2} / \mathscr{H}_{\infty}$ control design procedure is based explicitly on transfer function norms, which account for the $\boldsymbol{A}, \boldsymbol{B}$ and $\boldsymbol{C}$ matrices in the state-space form, whereas transient energy growth is related only to the eigenvectors and eigenvalues of $\boldsymbol{A}$. Non-normality is generally reduced by application of effective control feedback, but this is only a 'byproduct' of the modern control design, it is not its explicit 'target'. Analysing both transfer function norms and transient energy growth thus gives a more complete picture of the closed-loop system behaviour.

Finally, the results from tables 2 and 3 indicate that, for a given $\ell$ and $\alpha$, the transfer function norms and transient energy growth generally increase as the Reynolds number is increased, reflecting the increased sensitivity of the closed-loop system to disturbances and initial perturbations as the Reynolds number (and the number of open-loop unstable linear global modes) increases. A more detailed view of this behaviour is given in figure 4 , which illustrates the variation of the four tabulated performance indices for the uncontrolled $(R e<47, \square)$ and controlled $\left(R e>47, \mathscr{H}_{2}\right.$ full information control) linear wake model as a function of Reynolds number for two control penalties $\ell$. 


\subsection{2. $\mathscr{H}_{2}$ vs. $\mathscr{H}_{\infty}$ control designs: the linear trade-off}

Both in the case of full-information control and in the case estimator-based control, applying an $\mathscr{H}_{\infty}\left(\gamma_{0}\right)$ control design instead of an $\mathscr{H}_{2}$ control design was found to result in greater control energy (larger $\left\|\boldsymbol{T}_{u w}\right\|_{2}$ ) and a better worst-case disturbance rejection (smaller $\left\|\boldsymbol{T}_{x w}\right\|_{\infty}$ ). A robust controller essentially uses the additional control energy to ensure the constraint on the upper bound on the transfer function norm $\left\|\boldsymbol{T}_{z w}\right\|_{\infty}$ is enforced. This behaviour can result in either a better or worse Gaussian disturbance rejection (smaller or larger $\left\|\boldsymbol{T}_{x w}\right\|_{2}$, respectively) depending on the control penalty $\ell$. This is not surprising from a mathematical point of view since the robust control approach 'detunes' the corresponding optimal controller, implying that it increases the transfer function $\left\|\boldsymbol{T}_{z w}\right\|_{2}$. Note that, by (3.9), $\left\|\boldsymbol{T}_{z w}\right\|_{2}$ measures the response of both the state and the control to Gaussian disturbances. A balance between the two terms on the right-hand side of (3.9) implies that, as $\left\|\boldsymbol{T}_{z w}\right\|_{2}$ increases when $\gamma$ is decreased and the optimal controller is 'detuned', $\left\|\boldsymbol{T}_{x w}\right\|_{2}$ might either increase or decrease, as evidenced in tables 2 and 3.

At a given Reynolds number, the robustness parameter $\gamma$ in the $\mathscr{H}_{\infty}$ control design can be selected anywhere between $\gamma=\infty$ and $\gamma=\gamma_{0}$, which itself is a function of the two design parameters, $\gamma_{0}=\gamma_{0}(\ell, \alpha)$. When designing the controls, we must therefore perform, via appropriate selection of $\{\ell, \alpha, \gamma\}$, a trade-off between the average control energy consumed and the rejection of both Gaussian disturbances and worst-case disturbances; as mentioned previously, intermediate values of all three control design parameters are generally preferred. We now examine the behaviour of the closed-loop system as the value of $\gamma$ is varied over the admissible range $\left(\gamma_{0}, \infty\right)$.

Figure 5 displays numerical computations of transfer function norms and maximum transient energy growth for the controlled line wake model at $R e=175$ with $\mathscr{H}_{\infty}$ estimator-based control applied, taking $\ell=0.1$ and $\alpha=0.1$, as a function of the robustness parameter $\gamma$ on the interval $\left(\gamma_{0}, \infty\right)$. As seen in figure 5, the worst-case response of the state of the system (measured by $\left\|\boldsymbol{T}_{x w}\right\|_{\infty}$ ) decreases as $\gamma$ is reduced, in this case balanced by increases in both the average control energy used (measured by $\left.\left\|\boldsymbol{T}_{u w}\right\|_{2}\right)$ and the response of the state of the system to Gaussian disturbances (measured by $\left\|\boldsymbol{T}_{x w}\right\|_{2}$ ). The effect on transfer function norms as the scalar parameters are varied is found to be monotonic, but the effect on transient energy growth $E_{\max }$ is not. Note also that, when $\gamma$ approaches $\gamma_{0}$, a modest decrease in $\left\|\boldsymbol{T}_{x w}\right\|_{\infty}$ is accompanied by steep increases in both $\left\|\boldsymbol{T}_{\boldsymbol{u}}\right\|_{2}$ and $E_{\max }$. This property is, in fact, quite common in $\mathscr{H}_{\infty}$ control design, and was observed in several similar numerical simulations of the present system. As a consequence, when designing a robust controller, intermediate values for $\gamma$ are preferred; $\gamma=\gamma_{0}$ should not usually be selected.

In all cases considered, the performance of the system in the presence of Gaussian disturbances (as measured by $\left\|\boldsymbol{T}_{x w}\right\|_{2}$ and $\left\|\boldsymbol{T}_{u w}\right\|_{2}$ ) which was achieved by an $\mathscr{H}_{\infty}$ controller with a given penalty $\ell$ on the control could also be achieved (and even improved) using an $\mathscr{H}_{2}$ controller with a smaller penalty on the control. Even the values of $\left\|\boldsymbol{T}_{x w}\right\|_{\infty}$ achieved by the $\mathscr{H}_{\infty}$ control designs could be matched by the $\mathscr{H}_{2}$ controller simply by reducing $\ell$. Thus, no clear advantage of choosing an $\mathscr{H}_{\infty}$ controller over an $\mathscr{H}_{2}$ controller was evident from the linear analysis, even for intermediate values for $\gamma$. However, in $\S 5$, it is shown that, in fact, the $\mathscr{H}_{\infty}$ control designs, which target control effort specifically on the response to 'worst-case' disturbances, provided a tangible advantage over their $\mathscr{H}_{2}$ counterparts when applied to the full nonlinear system. 

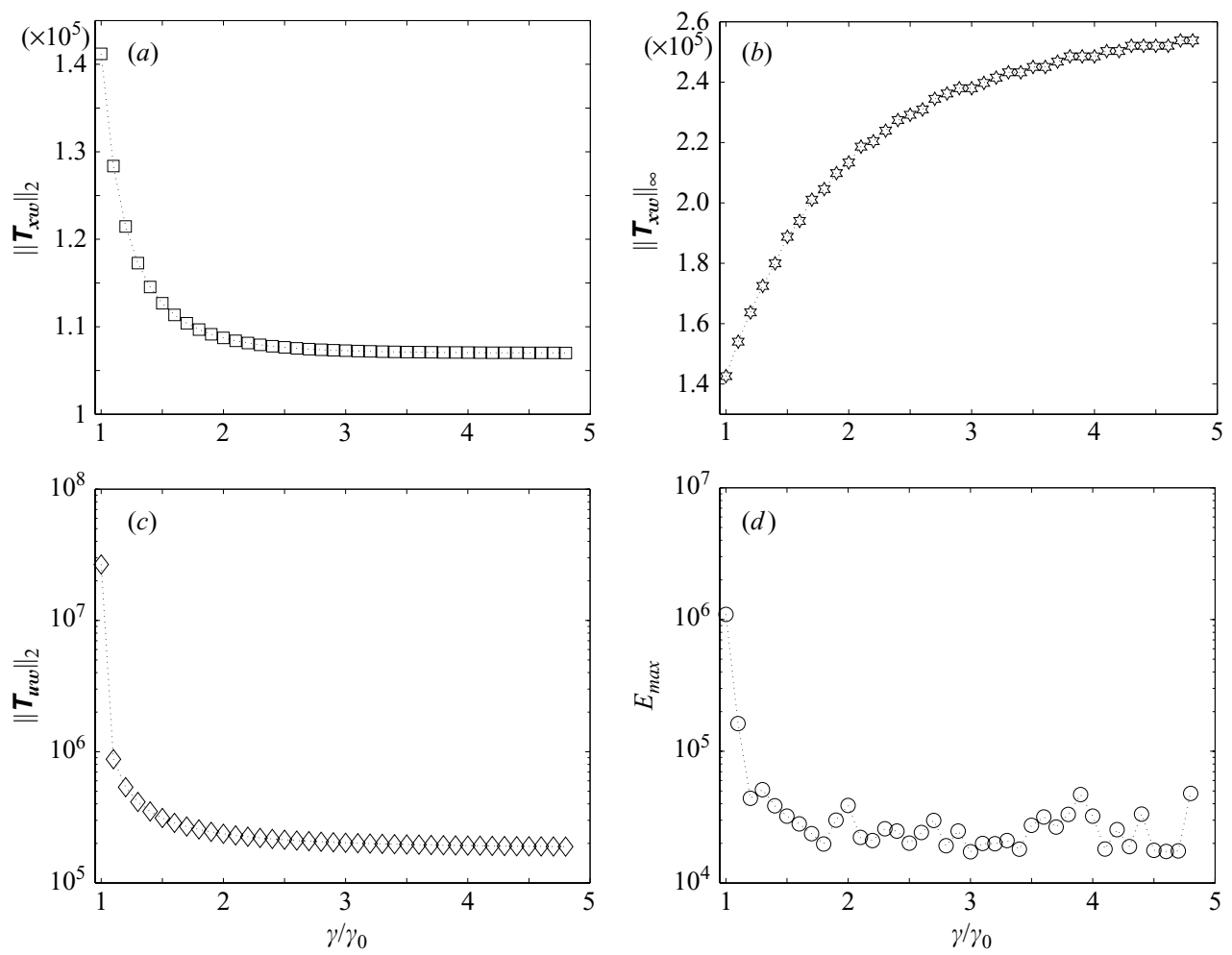

FIGURE 5. Performance and robustness characterizations for the controlled wake model at $R e=175$ with $\mathscr{H}_{\infty}$ estimator-based control applied $(\ell=0.1, \alpha=0.1)$ as a function of the robustness parameter $\gamma$, around $\gamma_{0}=130323$. (a) Transfer function 2-norm $\left\|\boldsymbol{T}_{x w}\right\|_{2} ;(b)$ transfer function $\infty$-norm $\left\|\boldsymbol{T}_{x w}\right\|_{\infty} ;(c)$ transfer function 2-norm $\left\|\boldsymbol{T}_{\boldsymbol{u}}\right\|_{2} ;(d)$ maximum transient energy growth $E_{\max }$. All plots are semilog-y.

\section{Control of the nonlinear wake model}

We now examine the possible application of the modern linear control designs derived and tested in the linear setting above to the fully nonlinear wake model (2.1). Note that the linear control designs described in the previous sections were based on linear analysis and, in general, we would not expect that they will be able to suppress the nonlinear global modes exhibited by the full nonlinear CGL system. Nevertheless, because of the simplicity and flexibility of the linear modern control design approach and the practical importance of the problem of wake stabilization, it is worth trying it anyway.

The simulations presented in the remainder of this work use the same spatial discretization scheme as described previously and a semi-implicit Adams-BashforthCrank-Nicholson (ABCN) scheme for time advancement, after one implicit Euler step to start the computation, and arbitrary finite-energy initial conditions. The system is marched in time without control until it reaches 'statistical steady state' (that is, until it approaches the limit cycle which forms the attractor of the nonlinear system). Control is then turned on in an attempt to stabilize the nonlinear system from an arbitrary position on its attractor using fixed-gain linear feedback. We will first discuss the case in which full state information is available, then discuss the construction of a nonlinear estimator with linear feedback based on the measurements (called an 

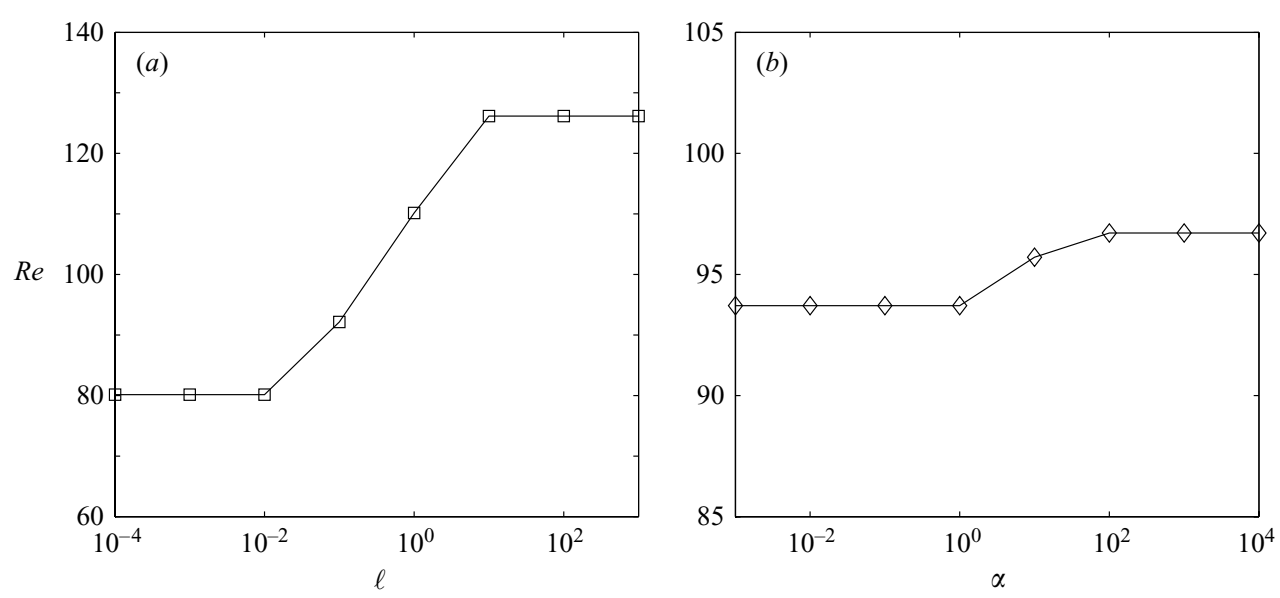

Figure 6. (a) Maximum Reynolds number for stability of the nonlinear wake model from the attracting limit cycle with full-information linear $\mathscr{H}_{2}$ control applied at $x_{f}=0$, as a function of $\ell$. (b) Maximum Reynolds number for convergence of the extended Kalman filter to the state of the nonlinear wake model with a single noisy sensor at $x_{s}=1.5$, as a function of $\alpha$.

extended Kalman filter when working with the $\mathscr{H}_{2}$ feedback gains), then synthesize the controller with the estimator.

\subsection{Full-information control of the nonlinear equation}

Using full state information, the control procedure descibed in $\S 33.3$ is now applied to the full CGL system (2.1) with the stabilizing cubic nonlinear term included. The continuous equation is discretized as before

$$
\frac{\partial \psi}{\partial t}=\mathscr{L} \psi-\eta|\psi|^{2} \psi+\delta\left(x-x_{f}\right) \boldsymbol{u}(t) \Rightarrow \dot{\boldsymbol{x}}=\boldsymbol{A} \boldsymbol{x}+\boldsymbol{B} \boldsymbol{u}-\eta|\boldsymbol{x}|^{2} \boldsymbol{x},
$$

with, again, linear feedback of the form $\boldsymbol{u}=\boldsymbol{K} \boldsymbol{x}$, with $\boldsymbol{K}$ determined from the solution $\boldsymbol{X}$ of the appropriate Riccati equation in (3.5); the adjustable design parameters are thus the same as described previously, the control penalty $\ell$ and the robustness parameter $\gamma$.

\subsubsection{Maximum Reynolds number for full-information stabilization of the nonlinear system}

For sufficiently small Reynolds number, it was found that the linear control feedback in the full-information setting was able to stabilize to zero the nonlinear system (that is, to drive $\|\psi\|$ to zero) from everywhere on the attracting limit cycle of the uncontrolled nonlinear system, as shown in figure 6(a). Curiously, as the penalty $\ell$ in the control formulation is increased, though the disturbance rejection of the controlled linear system degrades (i.e. $\left\|\boldsymbol{T}_{x w}\right\|_{2}$ increases), the maximum Reynolds number for which linear stabilization of the nonlinear system was observed improves. In other words, figure 6(a) reveals that increasing $\ell$ (reducing the energy of the control feedback) increases the maximum $R e$ for which the linear control feedback stabilizes the nonlinear system from its limit cycle when no external disturbance forcing is applied. Informally, we might say that, by reducing the energy of the control feedback, the tendency for the control itself to disrupt the system when flow perturbations are large (and thus the linearization of the system is not entirely valid) 

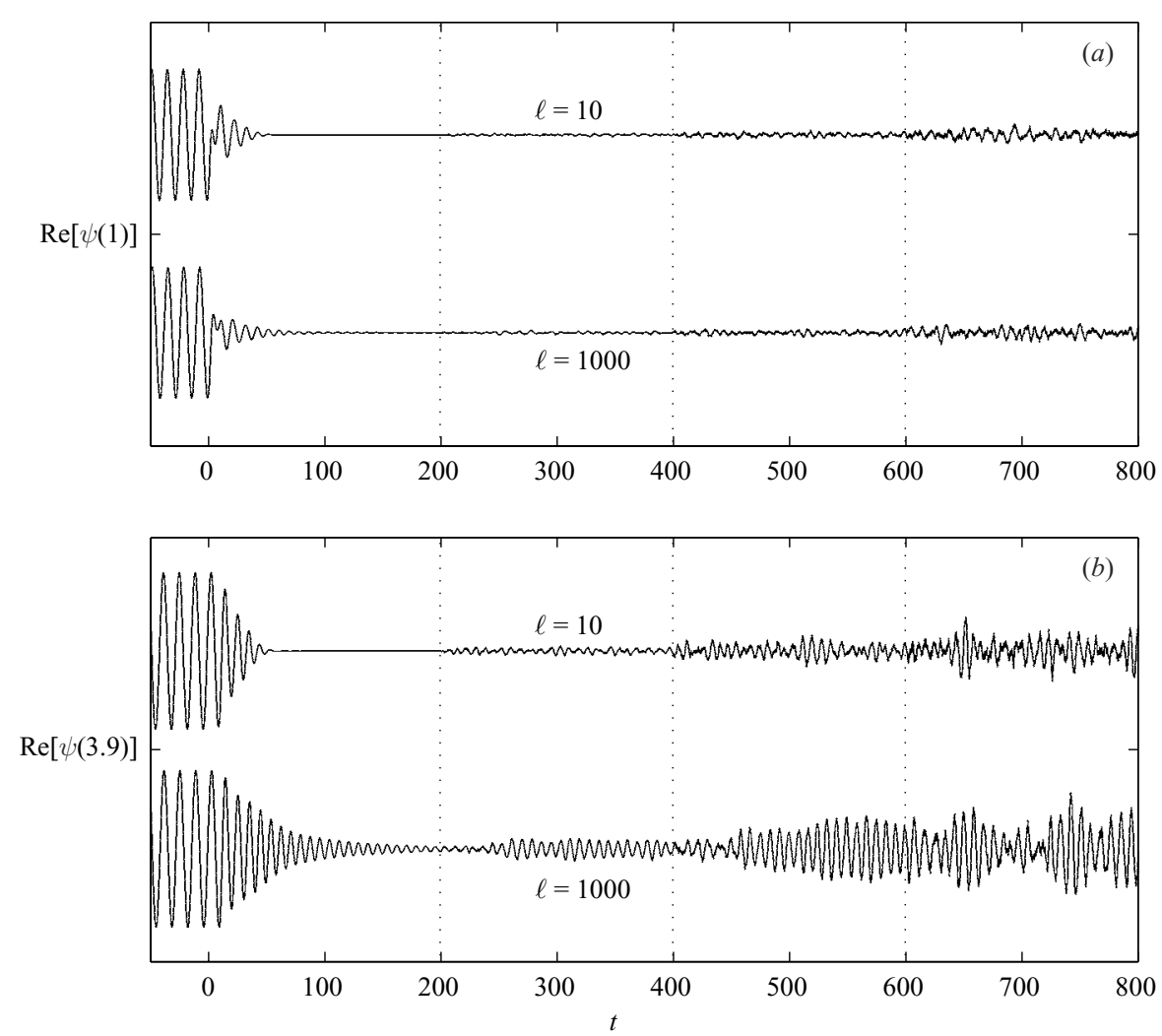

FIGURE 7. Comparison of the time simulation of the nonlinear Ginzburg-Landau equation for $R e=80$ with full information $\mathscr{H}_{2}$ control switched on at $t=0$ for two different control penalties and spatial locations; $(a)$ real part of $\psi(1) ;(b)$ real part of $\psi(3.9)$. The system is forced by Gaussian disturbances to the state equation of increasing intensity in time: $t=0 \rightarrow 200$ : no disturbances; $t=200 \rightarrow 400$ : disturbances of intensity $10 \% ; t=400 \rightarrow 600$ : disturbances of intensity $25 \% ; t=600 \rightarrow 800$ : disturbances of intensity $50 \%$.

is reduced. That is, strong control feedback computed using linear theory (with small $\ell$ ) will sometimes force the fully nonlinear system strongly in the wrong direction; weak control feedback (computed with large $\ell$ ) nudges the nonlinear system only gently and therefore has less of a tendency to drive it unstable.

\subsubsection{Noise rejection of the nonlinear closed-loop plant}

Since $\mathscr{H}_{2}$ controls with $10 \leqslant \ell<\infty$ are able to stabilize the full nonlinear system from the attracting limit cycle up to $R e \approx 125$, a secondary control design optimization criterion might be considered to determine the best value of $\ell$ in this range. As we have seen in the previous sections characterizing the control of the linear wake model, the transfer function norms from the noise to the state and the maximum transient energy growth are reduced when reduced values for $\ell$ are selected. The control design with $\ell=10$ (that is, the smallest value for $\ell$ at which it is possible to delay the instability up to $R e \approx 125$ ) might thus, in some sense, be considered favourable in terms of both stabilization and disturbance rejection in the full nonlinear system. To quantify this idea further, time-simulations of the closed-loop system at $R e=80$ (initialized on the attracting limit cycle) were performed and are reported in figure 7 for two different full-information control strategies (corresponding to $\ell=1000$ and 
$\ell=10$ ), with Gaussian disturbances applied to the state equation which increased in intensity over time, and the resulting system dynamics plotted at two spatial locations $(x=1$ and $x=3.9)$. For lack of a better non-dimensionalization, we define here the intensity of the external disturbances to the state equation as simply the ratio of the standard deviation of these disturbances to the amplitude of the oscillation of the attracting limit cycle in the uncontrolled system; with this definition, a noise of intensity $50 \%$ has a standard deviation equal to a half of the amplitude of the oscillations of the uncontrolled limit cycle.

The effect of the parameter $\ell$ on the control effectiveness is readily apparent in this test immediately after the control is turned on $\left(\right.$ at $\left.t=0^{+}\right)$, when the nonlinear system is stabilized from the attracting limit cycle of the uncontrolled system and no external disturbances are applied to the state equation. The oscillation close to the actuator (at $x=1$ ) is quickly subdued for the $\ell=10$ case, whereas for the $\ell=1000$ case small oscillations 'ring' for a considerable period of time. Perhaps even more remarkable is the observation that the oscillation far downstream (at $x=3.9$ ) is quickly subdued in the $\ell=10$ case as well; on the other hand, when less control feedback is used in the $\ell=1000$ case, the oscillations take a relatively long time (several periods) to decay, appearing to be relatively poorly damped.

The effect of the parameter $\ell$ on the control effectiveness is also apparent in the disturbance rejection characteristics of the nonlinear system. We observe in figure 7 that close to the actuator (at $x=1$ ), the two controls have roughly the same disturbance rejection characteristics. On the other hand, farther downstream (at $x=3.9$ ), the $\ell=10$ case appears to be significantly more effective at rejecting disturbances.

Finally, it is significant to notice that downstream of the actuator (at $x=3.9$ ) in the case $\ell=1000$, in which a relatively small amount of control feedback is applied, a specific frequency is preferentially excited by the Gaussian system disturbances (see the bottom half of figure $7 b$ ). This is a characteristic of what was referred to in the introduction as a 'slightly damped oscillator', in which at least one global mode of the system is only marginally stabilized.

\subsection{3. $\mathscr{H}_{2}$ vs. $\mathscr{H}_{\infty}$ control designs: the nonlinear trade-off}

As seen in figure 6(a), the maximum Reynolds number for $\mathscr{H}_{2}$ stabilization of the present nonlinear CGL system from the attracting limit cycle using full state information is $R e \approx 125$. By tuning the parameter $\gamma$ in the $\mathscr{H}_{\infty}$ control design in our numerical tests, this value could not be increased further.

To quantify disturbance rejection characteristics of the nonlinear closed-loop plant with $\mathscr{H}_{\infty}$ control applied, we repeated the simulations of figure 7 (at $R e=80$ ) using full-information $\mathscr{H}_{\infty}$ control with the smallest value of $\gamma$ which stabilized the system in the nonlinear setting, termed hereinafter $\gamma_{0}^{n l}=\gamma_{0}^{n l}(\ell)$; the result is shown in figure 8. Of course, in all cases it was found that $\gamma_{0}^{n l}>\gamma_{0}$. It is seen that, close to the actuator $(x=1)$, the results are quite similar to the $\mathscr{H}_{2}$ case. Farther downstream of the actuator $(x=3.9)$, the $\mathscr{H}_{\infty}\left(\gamma_{0}^{n l}\right)$ case for $\ell=1000$ appears to reject large disturbances (on $t \in[600,800]$ ) slightly better than the $\mathscr{H}_{2}$ case with $\ell=1000$, but not better than the $\mathscr{H}_{2}$ case with $\ell=10$. From these observations, no tangible advantage of the $\mathscr{H}_{\infty}$ control design strategy over the $\mathscr{H}_{2}$ control design strategy is readily apparent in the present system at $R e=80$.

By performing a large number of numerical simulations of the present system at $R e>80$ with increasing noise strength, in the same fashion as figures 7 and 8 , the advantages of the $\mathscr{H}_{\infty}$ control design strategy finally became evident. In particular, 

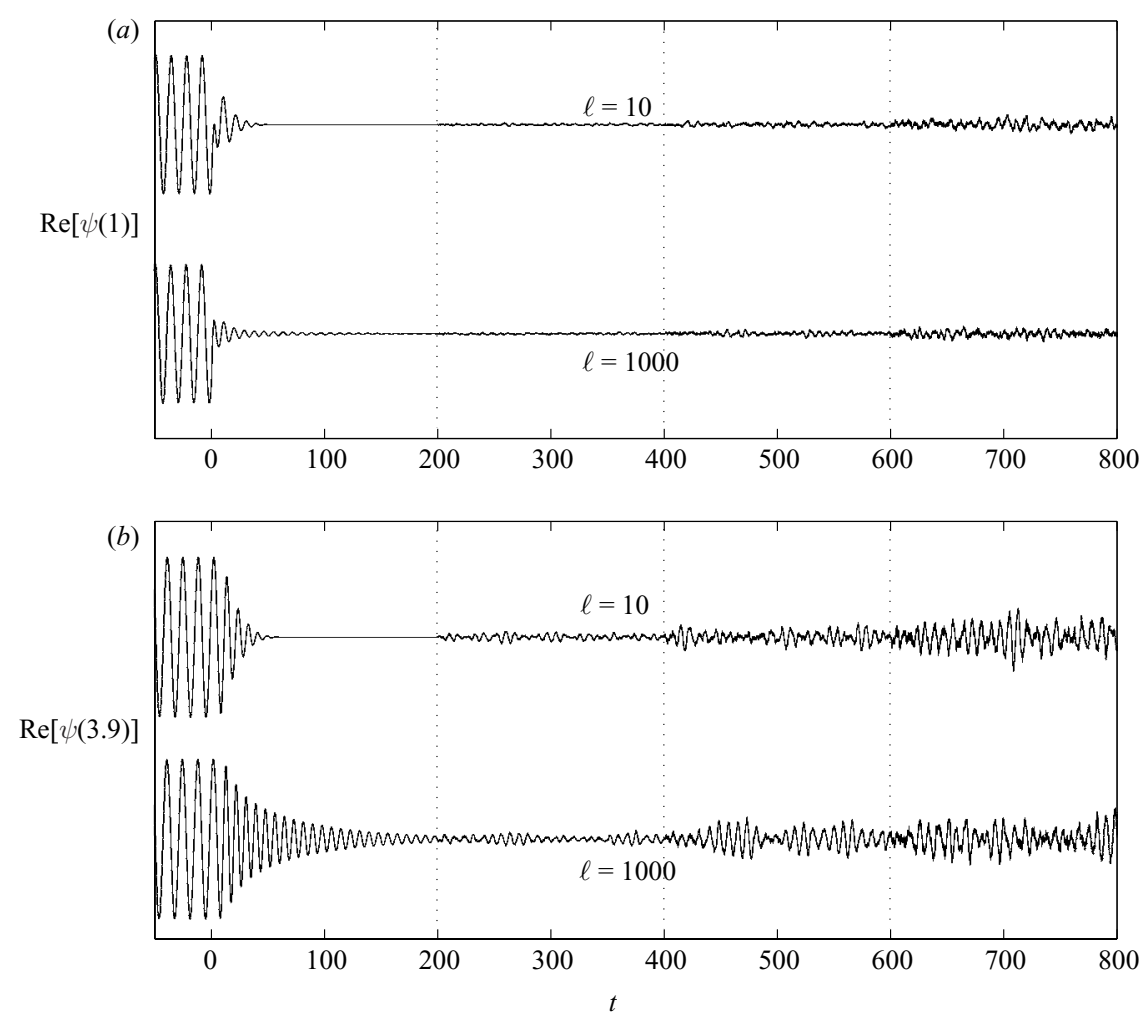

FIGURE 8. Comparison of the time simulation of the nonlinear Ginzburg-Landau equation for $R e=80$ with full information $\mathscr{H}_{\infty}$ control switched on at $t=0$ for two different control penalties and spatial locations, and the smallest value $\gamma_{0}^{n l}$ for the robustness parameter $\gamma$; (a) real part of $\psi(1)$; (b) real part of $\psi(3.9)$. The system is forced by Gaussian noise of increasing strength in time: $t=0 \rightarrow 200$ : no noise; $t=200 \rightarrow 400$ : noise of intensity $10 \%$; $t=400 \rightarrow 600:$ noise of intensity $25 \% ; t=600 \rightarrow 800$ : noise of intensity $50 \%$.

below $R e \approx 99$, the Gaussian disturbance rejection of the closed-loop plant obtained by decreasing $\ell$ to the smallest value for which stabilization of the nonlinear system from the attracting limit cycle occurred (see figure $6 a$ ) was systematically better than the disturbance rejection given by decreasing $\gamma$ for increased values of $\ell$ (this is true, for example, in the cases illustrated in figures 7 and 8). However, for $R e>99$, the opposite behaviour was observed, thus tipping the scales in favour of the $\mathscr{H}_{\infty}$ control designs at higher Reynolds numbers.

Finally, in addition to stabilization and Gaussian disturbance rejection, the third aspect of the nonlinear control design trade-off concerns worst-case disturbance rejection. To characterize this, we denote by $\widetilde{\gamma}_{0}^{n l}$ the smallest possible value of $\gamma$ for which a linear $\mathscr{H}_{\infty}$ control design is capable of stabilizing the nonlinear wake model at a particular value of the Reynolds number with disturbances applied which are continuously equal to the linear worst-case disturbance forcing. Mathematically, this corresponds to adding a $\boldsymbol{G}_{1} \tilde{\boldsymbol{w}}_{1}$ contribution to the state equation (5.1), with the disturbance $\tilde{\boldsymbol{w}}_{1}$ chosen to be exactly that which locally maximizes the quadratic cost function in the linear setting (Doyle et al. 1989):

$$
\tilde{\boldsymbol{w}}_{1}=\frac{1}{\gamma^{2}} \mathbf{S}_{1}^{-1} \mathbf{G}_{1}^{*} \boldsymbol{X} \boldsymbol{x} .
$$


This assumption is a mathematical statement of a sort of worst-case scenario. It is important to note that, from a linear standpoint, the linear wake model with this worst-case disturbance continuously added to the system is stable (Doyle et al. 1989), as $\mathscr{H}_{\infty}$ control theory explicitly accounts for this disturbance in the control design. As a consequence, $\widetilde{\gamma}_{0}^{n l} \geqslant \gamma_{0}$.

We performed several numerical simulations of the nonlinear model with both the above worst-case disturbance continuously fed into the system and additional Gaussian disturbances added to the resulting system. In the same fashion as in figure 7, the observation of the Gaussian disturbance rejection properties with increasing disturbance strength led to the following conclusions. Below $\operatorname{Re} \approx 84$, there is sufficient flexibility in the $\mathscr{H}_{2}$ control design to stabilize the nonlinear system from the attracting limit cycle of the uncontrolled system while achieving both superior Gaussian and worst-case disturbance rejection than for the $\mathscr{H}_{\infty}$ control designs. However, for $R e>84$, the $\mathscr{H}_{\infty}\left(\widetilde{\gamma}_{0}^{n l}\right)$ controller had superior worst-case disturbance rejection characteristics. Thus, when designing for the worst-case disturbance scenario at $\operatorname{Re}>84, \mathscr{H}_{\infty}\left(\widetilde{\gamma}_{0}^{n l}\right)$ control designs are generally preferred over $\mathscr{H}_{2}$ control designs. Note that $R e=84$ corresponds to the appearance of a second unstable linear global mode in the system.

\subsection{Estimation of the nonlinear equation}

We now consider the problem of state estimation of the nonlinear CGL system. There is broad experience in the field of nonlinear estimation that one of the most effective techniques available is the use of an extended Kalman filter (see, e.g. Grewal $\&$ Andrews 1993) or its $\mathscr{H}_{\infty}$ counterpart, incorporating the system nonlinearity into the estimator model. Following this approach, the equations for the evolution of the state $\boldsymbol{x}$ and the state estimate $\hat{\boldsymbol{x}}$ (in the unforced, undisturbed case) are

$$
\left.\begin{array}{ll}
\dot{\boldsymbol{x}}=\boldsymbol{A} \boldsymbol{x}-\eta|\boldsymbol{x}|^{2} \boldsymbol{x}, & \boldsymbol{y}=\boldsymbol{C} \boldsymbol{x}, \\
\dot{\hat{\boldsymbol{x}}}=\boldsymbol{A} \hat{\boldsymbol{x}}-\eta|\hat{\boldsymbol{x}}|^{2} \hat{\boldsymbol{x}}+\boldsymbol{v}, & \hat{\boldsymbol{y}}=\boldsymbol{C} \hat{\boldsymbol{x}},
\end{array}\right\}
$$

with the measurement feedback of the form $\boldsymbol{v}=\boldsymbol{L}(\boldsymbol{y}-\hat{\boldsymbol{y}})$, with $\boldsymbol{L}$ determined from the solution $\boldsymbol{Y}$ of the appropriate Riccati equation in 3.5.

\subsubsection{Maximum Reynolds number for stabilization of the nonlinear estimation error}

For sufficiently small Reynolds number, it was found that the linear measurement feedback in the estimator was able to stabilize to zero the estimation error of the nonlinear system from everywhere on the attracting limit cycle of the system, as shown in figure $6(b)$. As the penalty $\alpha$ in the control formulation is decreased, the maximum Reynolds number for which this degree of stabilization in the estimator is possible also decreases, but only mildly.

The efficiency of the extended Kalman filtering technique on the nonlinear system is illustrated in figure 9 at $R e=80(x=3.9, \alpha=10)$ where the estimation feedback is switched on at $t=0$, in the presence of mild Gaussian disturbances. The estimation error is approximately constant for $t<0$ (reflecting an out of phase relationship between the state and the state estimate) and is seen to be driven to zero rapidly when $t>0$.

\subsubsection{Disturbance rejection of the extended Kalman filter}

Numerical simulations of the equations governing the nonlinear wake model and its estimate showed the perhaps surprising result that the noise-to-disturbance ratio $\alpha$ has apparently little effect on the disturbance rejection properties of the estimated plant in 

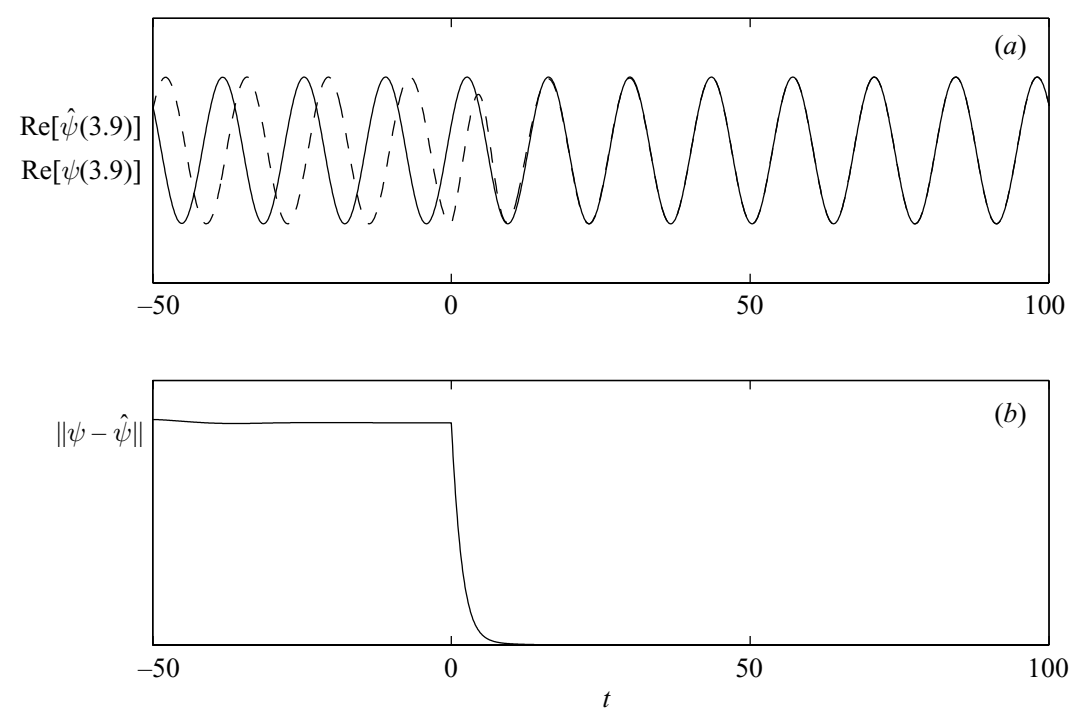

FIGURE 9. Evolution of the nonlinear Ginzburg-Landau system and its estimate for $R e=80$ under $1 \%$ Gaussian disturbance conditions with $\mathscr{H}_{2}$ estimation (computed for $\alpha=10$ ) switched on at $t=0$ and initial conditions on the attracting limit cycles of (5.3). (a) Real part of the state $\psi(3.9)$ (solid) and the state estimate $\hat{\psi}(3.9)$ (dashed); (b) energy of the estimation error.

the nonlinear setting. This observation, which applied for both Gaussian disturbances and worst-case disturbances both near the actuator and farther downstream, is in sharp contrast with the observations in the linear setting, where it was found that $\alpha$ had a major influence on the values of linear transfer function norms of the closedloop system.

\subsection{Synthesis: the nonlinear compensator}

As in the linear case, the final step in the control design is to build a nonlinear compensator for the nonlinear system by combining the estimation and control strategies described in the two previous sections. The state equation for the nonlinear evolution of $\boldsymbol{x}$ is given by (5.1) and the corresponding estimator equation for the nonlinear evolution of $\hat{\boldsymbol{x}}$ is given in (5.3), with an additional term added to the righthand side account for the control input $\boldsymbol{u}$ as in (5.1). The feedback into the estimator is taken to be the linear form $\boldsymbol{v}=\boldsymbol{L}(\boldsymbol{y}-\hat{\boldsymbol{y}})$ as before, and the control feedback is based linearly on the state estimate such that $\boldsymbol{u}=\boldsymbol{K} \hat{\boldsymbol{x}}$. The three scalar control design parameters are the same as in the linear study, $(\ell, \alpha, \gamma)$.

\subsubsection{Maximum Reynolds number for measurement-based stabilization of the system}

The effectiveness of the nonlinear compensator described above (based on a single noisy measurement at $x_{s}=1.5$ ) on the nonlinear CGL system is illustrated in figure 10 at $R e=80$ at $x=1$ for $\ell=10$ and $\alpha=10$. The control is switched on at $t=0$ and rapidly drives both the estimation error and the state to zero.

The maximum Reynolds number for stabilization of the present system by this nonlinear compensator from anywhere on the attracting limit cycle of the uncontrolled system was found to coincide with the information in figures 6(a) and 6(b). Specifically, for a particular $\ell$ and $\alpha$, the nonlinear compensator could stabilize the nonlinear system from anywhere on the attracting limit cycle at a Reynolds number less than both the maximum Reynolds number for linear stabilization of the nonlinear system 

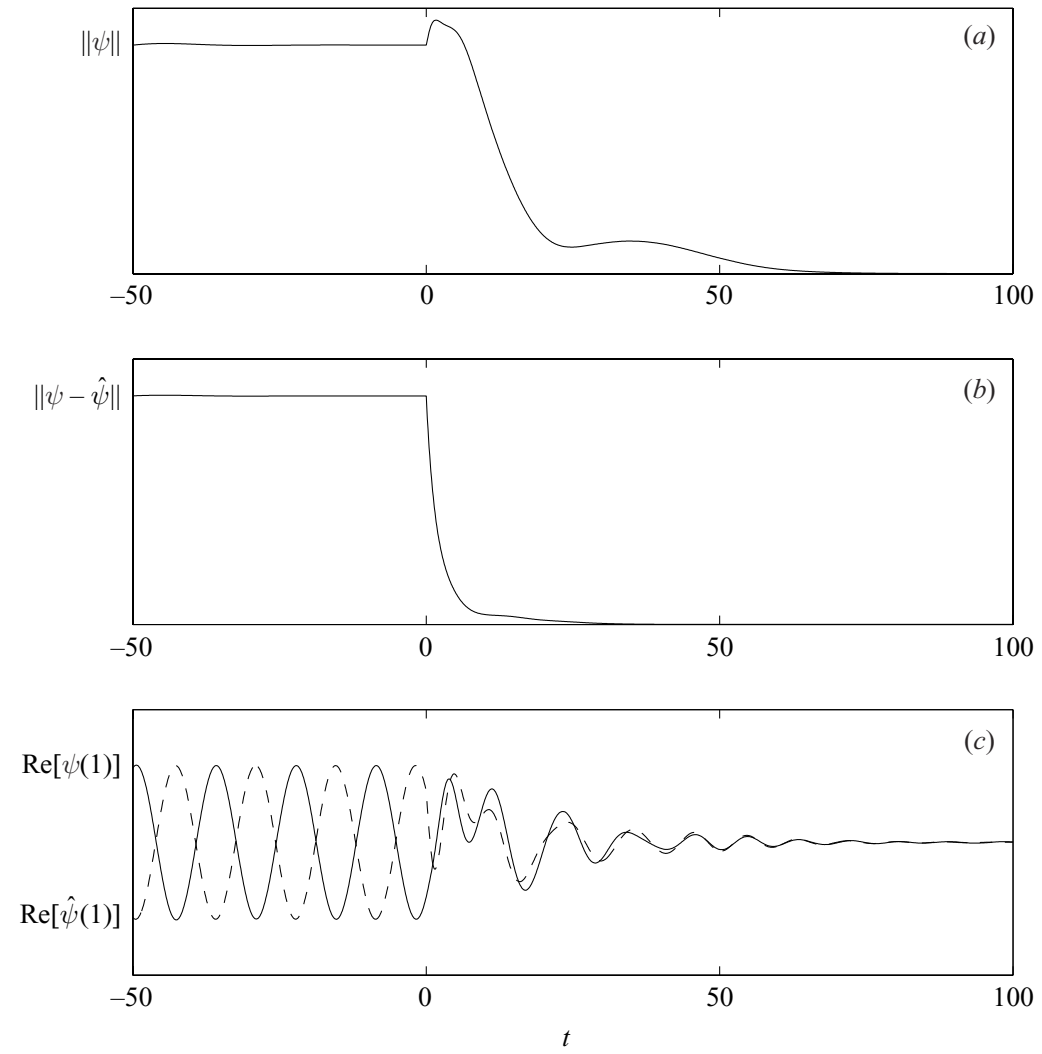

FiguRE 10. Time simulation of the nonlinear Ginzburg-Landau equation for $R e=80$ under $1 \%$ Gaussian noise conditions with an $\mathscr{H}_{2}$ compensator (computed for $\ell=10$ and $\alpha=10$ ) switched on at $t=0$ and arbitrary initial conditions. (a) Energy of the state $\psi ;(b)$ energy of the estimation error $\psi-\hat{\psi} ;(c)$ real part of $\psi(1)$ (solid) and $\hat{\psi}(1)$ (dashed).

with full information (as depicted in figure $6 a$ ), and the maximum Reynolds number for stabilization of the estimation error (as depicted in figure $6 b$ ). Thus, the maximum achievable Reynolds number for stability of the nonlinear system from anywhere on the attracting limit cycle of the uncontrolled nonlinear system based on a single noisy measurement at $x_{s}=1.5$ is $R e \approx 97$. For comparison, the proportional control scheme proposed in RM96 is able to suppress the nonlinear oscillations in the same system, using the same actuator/sensor configuration, only up to a Reynolds number of $R e \approx 64$.

\subsubsection{Disturbance rejection properties of the compensator}

Numerical simulations of the nonlinear system with the nonlinear compensator described above applied (in the same fashion as the simulations presented in figures 7 and 8) confirmed that the disturbance rejection characteristics of the closed-loop nonlinear system depend most strongly on the control parameter $\ell$ in addition to the Reynolds number Re. As noted in $\S 5.2, \alpha$ has apparently only a weak effect on these properties in the nonlinear setting.

\section{Discussion and conclusion}

This paper addressed the effectiveness of modern $\mathscr{H}_{2}$ and $\mathscr{H}_{\infty}$ control theory applied to a one-dimensional PDE model of spatially developing flow systems. This 
model has been thoroughly benchmarked in previous studies and has proved to capture well the important hydrodynamic features of such systems. We thus expect that both the qualitative insight gained and quantitative control strategies derived will carry over fairly directly to applications in several physical spatially developing shear flow systems of important engineering relevance. Such extensions of this approach to experimental rigs, such as that discussed in Smith, Siegel \& McLaughlin (2002), will be explored in future work.

As previous investigations had shown that simple proportional feedback strategies were only effective up to a relatively low Reynolds number in the present system (even in the linear setting), a dynamic compensator was designed in this study using modern control theory. It was shown that the compensators so designed lead to substantially better performance on the nonlinear CGL system than the proportional feedback strategies proposed previously.

In the linear setting, we have shown in prior work (Lauga \& Bewley 2003) that modern control strategies can, in theory, stabilize the present system at any Reynolds number with a single actuator and a single sensor if sufficiently high numerical precision is used in the control derivation. However, owing to the diminishing controllability and observability of the open-loop unstable modes as the Reynolds number is increased, this theoretical result is not useful in practice, and performance characterizations (such as transfer function norms and maximum transient energy growth) which quantify the effect of non-normality in the closed-loop system are necessary to design and characterize effective controls that actually work in the nonlinear setting.

At relatively low Reynolds numbers $(47<R e<84)$, it was found that $\mathscr{H}_{2}$ control designs (that is, with $\gamma=\infty$ ) with an intermediate penalty on the control $\ell=10$ gave, by some measure, the best overall performance. The effect of the full-information linear control scheme on the nonlinear equation was also tested, and it was shown that full-information-based linear control is effective in subduing the nonlinear system initialized anywhere on the attracting limit cycle of the uncontrolled system up to $R e \approx 125$. A study of the effectiveness of the linear control in rejecting disturbances in the nonlinear CGL system indicated that for $R e>84$, the full-information $\mathscr{H}_{\infty}$ control designs achieved a better worst-case noise rejection than the corresponding $\mathscr{H}_{2}$ control designs, and for $R e>99$, the full-information $\mathscr{H}_{\infty}$ control designs achieved better Gaussian noise rejection than the corresponding $\mathscr{H}_{2}$ control designs. An extended Kalman filter was also tested for estimating the nonlinear CGL system, and it was shown that such a filter is capable of driving the estimation error to zero up to $R e \approx 97$. Finally, when combining the linear controller with the extended Kalman filter, the resulting compensator was shown to be effective in stabilizing the nonlinear CGL system from anywhere on the attracting limit cycle of the uncontrolled nonlinear system with a single actuator/sensor pair up to $R e \approx 97$.

One of the conclusions from Monkewitz (1989) and Huerre \& Monkewitz (1990) concerning the control of open flows was that it was very likely that each linear global mode needed to be stabilized by a separate actuator/sensor pair. The present paper, together with Lauga \& Bewley (2003), has shown that, with the proper control algorithm, this is, in fact, not the case. It has been shown that, with an appropriate feedback algorithm, we can indeed linearly stabilize several open-loop unstable modes with only a single actuator/sensor pair and time-independent control feedback gains. However, as the Reynolds number is increased, the performance of the linear control design degrades to the point that they become ineffective on the full nonlinear system. In this case, multiple sensors and/or multiple actuators should be used to improve the controllability and observability of the open-loop unstable modes of the system, 
as proposed by Monkewitz (1989) and discussed further in Lauga \& Bewley (2003). Another issue of practical interest is the capability of control strategies designed at one Reynolds number to perform adequately at another Reynolds number (at so-called 'off-design' conditions). Such questions, as well as practical implementation issues, will be addressed in future work.

The authors gratefully acknowledge the encouragement and technical advise of Patrick Huerre and Jean-Marc Chomaz, many fruitful discussions with Robert Bitmead, Peter Schmid, Yuji Suzuki, Carlo Cossu, Markus Högberg, François Gallaire, and helpful references by Peter Monkewitz. The generous funding of CTE, Ecole des Mines de Paris and AFOSR (Belinda King) are also gratefully acknowledged.

\section{REFERENCES}

Aamo, O. M. \& Krstić, M. 2003 Flow Control by Feedback: Stabilization and Mixing. Springer.

Bender, C. M. \& Orszag, S. A. 1978 Advanced Mathematical Methods for Scientists and Engineers. McGraw-Hill.

BERGER, E. 1967 Suppression of vortex shedding and turbulence behind oscillating cylinders. Phys. Fluids 10, S191-S193.

Bers, A. 1983 Basic Plasma Physics I. North-Holland.

BEwLEY, T. R. 1999 Linear control and estimation of nonlinear chaotic convection: harnessing the butterfly effect. Phys. Fluids 11, 1169-1186.

Bewley, T. R. \& LiU, S. 1998 Optimal and robust control and estimation of linear paths to transition. J. Fluid Mech. 365, 305-349.

BriggS, R. J. 1964 Electron-Stream Interactions with Plasmas. MIT Press.

Chomaz, J. M., Huerre, P. \& Redekopp, L. G. 1987 Models of hydrodynamics resonances in separated shear flows. Proc. 6th Symp. on Turbulent Shear Flows, Toulouse, pp. 321-326.

Chomaz, J. M., Huerre, P. \& Redekopp, L. G. 1988 Bifurcations to local and global modes in spatially developing flows. Phys. Rev. Lett. 60, 25-28.

Chomaz, J. M., Huerre, P. \& Redekopp, L. G. 1991 A frequency selection criterion in spatially developing flows. Stud. App. Maths 84, 119-144.

Couarron, A. \& Chomaz, J. M. 1999 Fully nonlinear global modes in slowly varying flows. Phys. Fluids 12, 3688-3703.

Doyle, J. C., Glover, K., Khargonekar, P. P. \& Francis, B. A. 1989 State-space solutions to standard $\mathscr{H}_{2}$ and $\mathscr{H}_{\infty}$ control problems. IEEE Trans. Autonat. Control 34, 831-847.

Ezal, K., Pan, Z. \& Koкотоví́, P. V. 2000 Locally optimal and robust backstepping design. IEEE Trans. Autonat. Control 45, 260-271.

Ffowcs Williams, J. E. \& Zhao, B. C. 1989 The active control of vortex shedding. J. Fluids Struct. 3, 115-122.

Gaster, M. 1968 Growth of disturbances in both space and time. Phys. Fluids 11, 723-27.

Gillies, E. A. 1998 Low-dimensional control of the circular cylinder wake. J. Fluid Mech. 371, $157-178$

Green, M. \& Limebeer, D. J. N. 1995 Linear Robust Control. Prentice Hall.

Grewal, M. S. \& Andrews, A. P. 1993 Kalman Filtering Theory and Practice. Prentice Hall.

Hammond, D. A. \& Redekopp, L. G. 1997 Global dynamics of symmetric and asymmmetric wakes. J. Fluid Mech. 331, 231-260.

Högberg, M., Bewley, T. R. \& Henningson, D. S. 2003 Relaminarization of $R e_{\tau}=100$ turbulence using gain scheduling and linear state-feedback control. Phys. Fluids 15, 3572-3575.

Huerre, P. \& Monkewitz, P. A. 1985 Absolute and convective instabilities in free shear layers. J. Fluid Mech. 159, 151-168.

Huerre, P. \& Monkewitz, P. A. 1990 Local and global instabilities in spatially developing flows. Annu. Rev. Fluid. Mech. 22, 473-537.

Huerre, P. \& Rossi, M. 1998 Hydrodynamic instabilities in open flows. In Hydrodynamics and Nonlinear Instabilities (ed. C. Godreche \& P. Manneville), pp. 81-294. Cambridge University Press. 
LAUB, A. J. 1991 Invariant subspace methods for the numerical solution of Riccati equations. In The Riccati Equation (ed. S. Bittaini, A. J. Laub \& J. C. Willems), pp. 163-196. Springer.

Lauga, E. \& Bewley, T. R. 2003 The decay of stabilizability with Reynolds number in a linear model of spatially developing flows. Proc. R. Soc. 459, 2077-2095.

Le Gal, P., Ravoux, J. F., Floriani, E. \& DE Wit, T. D. 2003 Recovering coefficients of the complex Ginzburg-Landau equation from experimental spatio-temporal data: two examples from hydrodynamics. Physica D 174, 114-133.

Mathis, M., Provansal, M. \& Boyer, L. 1984 The Bénard-von Kármán instability: an experimental study near the threshold. J. Physique Lett. 45, 483-491.

Min, C. \& CHOI, H. 1999 Suboptimal feedback control of vortex shedding at low Reynolds numbers. J. Fluid Mech. 401, 123-156.

Monkewitz, P. A. 1988 The absolute and convective nature of instability in two-dimensional wakes at low Reynolds number. Phys. Fluids 31, 999-1006.

Monkewitz, P. A. 1989 Feedback control of global oscillations in fluid systems. AIAA Paper 89-0991.

Monkewitz, P. A. 1990 The role of absolute and convective instability in predicting the behaviour of fluid systems. Eur. J. Mech. B/Fluids 10, 395-413.

Monkewitz, P. A. 1993 Wake control. In Bluff-Body Wakes, Dynamics and Instabilities (ed. H. Eckelmann, A. L. Graham, P. Huerre \& P. A. Monkewitz), pp. 227-290. Springer.

Monkewitz, P. A., Huerre, P. \& Chomaz, J. M. 1993 Global linear stability analysis of weakly non-parallel shear flows. J. Fluid Mech. 251, 1-20.

Newell, A. C. \& Whitehead, J. A. 1969 Finite bandwidth, finite amplitude convection. J. Fluid Mech. 38, 279-303.

PARK, D. S. 1994 Theoretical analysis of feedback control of von Kármán vortex street at slightly supercritical Reynolds number. Eur. J. Mech. B/Fluids 13, 387-399.

PARK, D. S., LADD, D. M. \& Hendricks, E. W. 1993 Feedback control of a global mode in spatially developing flows. Phys. Lett. A 182, 244-248.

PARK, D. S., LAdD, D. M. \& Hendricks, E. W. 1994 Feedback control of von Kármán vortex shedding behind a circuler cylinder at low Reynolds numbers. Phys. Fluids 6, 2390-2405.

PIER, B. 2002 On the frequency selection of finite-amplitude vortex shedding in the cylinder wake. J. Fluid Mech. 458, 407-417.

Pier, B. \& Huerre, P. 2001 Nonlinear self-sustained structures and fronts in spatially developing wake flows. J. Fluid Mech. 435, 145-174.

Pier, B., Huerre, P., Chomaz, J. M. \& Couairon, A. 1998 Steep nonlinear gobal modes in spatially developing media. Phys. Fluids 10, 2433-2435.

Provansal, M., Mathis, M. \& Boyer, L. 1987 Bénard-von Kármán instability: transient and forced regimes. J. Fluid Mech. 182, 1-22.

Roussopoulos, K. 1993 Feedback control of vortex shedding at low Reynolds numbers. J. Fluid Mech. 248, 267-296.

Roussopoulos, K. \& Monkewitz, P. A. 1996 Nonlinear modelling of vortex shedding control in cylinder wakes. Physica D 97, 264-273.

Schmid, P. J. \& Henningson, D. S. 2001 Stability and Transition in Shear Flows. Springer.

Schumm, M., Berger, E. \& Monkewitz, P. A. 1994 Self-excited oscillations in the wake of two-dimensional bluff bodies and their control. J. Fluid Mech. 271, 17-53.

Smith, D. R., Siegel, S. \& McLaughlin, T. 2002 Modeling of the wake behind a circular cylinder undergoing rotational oscillation. AIAA Paper 2002-3066.

Stewartson, K. \& Stuart, J. T. 1971 A non-linear instability theory for a wave system in plane Poiseuille flow. J. Fluid Mech. 48, 529-545.

Williamson, C. H. K. 1996 Vortex dynamics in the cylinder wake. Annu. Rev. Fluid. Mech. 28, 477-539.

Zhou, K. \& Doyle, J. C. 1997 Essentials of Robust Control. Prentice Hall. 